\title{
Galaxy growth in a massive halo in the first billion years of cosmic history
}

D. P. Marrone ${ }^{1}$, J. S. Spilker ${ }^{1}$, C. C. Hayward ${ }^{2,3}$, J. D. Vieira ${ }^{4}$, M. Aravena ${ }^{5}$, M. L. N. Ashby ${ }^{3}$, M. B. Bayliss ${ }^{6}$, M. Béthermin 7 , M. Brodwin ${ }^{8}$, M. S. Bothwell ${ }^{9,10}$, J. E. Carlstrom ${ }^{11,12,13,14}$, S. C. Chapman $^{15}$, Chian-Chou Chen ${ }^{16}$, T. M. Crawford ${ }^{11,14}$, D. J. M. Cunningham ${ }^{15,17}$, C. De Breuck ${ }^{16}$, C. D. Fassnacht ${ }^{18}$, A. H. Gonzalez ${ }^{19}$, T. R. Greve ${ }^{20}$, Y. D. Hezaveh ${ }^{21,28}$, K. Lacaille ${ }^{22}$, K. C. Litke ${ }^{1}$, S. Lower ${ }^{4}$, J. Ma ${ }^{19}$, M. Malkan ${ }^{23}$, T. B. Miller ${ }^{15}$, W. R. Morningstar ${ }^{21}$, E. J. Murphy ${ }^{24}$, D. Narayanan ${ }^{19}$, K. A. Phadke ${ }^{4}$, K. M. Rotermund ${ }^{15}$, J. Sreevani ${ }^{4}$, B. Stalder ${ }^{25}$, A. A. Stark ${ }^{3}$, M. L. Strandet ${ }^{26,27}$, M. Tang ${ }^{1}, \&$ A. Weiß ${ }^{26}$

${ }^{1}$ Steward Observatory, University of Arizona, 933 North Cherry Avenue, Tucson, AZ 85721, USA

${ }^{2}$ Center for Computational Astrophysics, Flatiron Institute, 162 Fifth Avenue, New York, NY 10010, USA

${ }^{3}$ Harvard-Smithsonian Center for Astrophysics, 60 Garden Street, Cambridge, MA 02138, USA

${ }^{4}$ Department of Astronomy, University of Illinois, 1002 West Green St., Urbana, IL 61801

${ }^{5}$ Núcleo de Astronomía, Facultad de Ingeniería, Universidad Diego Portales, Avenida Ejército 441, Santiago, Chile

${ }^{6}$ Kavli Institute for Astrophysics \& Space Research, Massachusetts Institute of Technology, 77 Massachusetts Avenue, Cambridge, MA 02139, USA

${ }^{7}$ Aix Marseille Université, CNRS, LAM, Laboratoire d'Astrophysique de Marseille, Marseille, France

${ }^{8}$ Department of Physics and Astronomy, University of Missouri, 5110 Rockhill Road, Kansas City, MO 64110, USA

${ }^{9}$ Cavendish Laboratory, University of Cambridge, 19 J.J. Thomson Avenue, Cambridge, CB3 OHE, $U K$

${ }^{10}$ Kavli Institute for Cosmology, University of Cambridge, Madingley Road, Cambridge CB3 OHA, UK

${ }^{11}$ Kavli Institute for Cosmological Physics, University of Chicago, 5640 South Ellis Avenue, Chicago, IL 60637, USA

${ }^{12}$ Department of Physics, University of Chicago, 5640 South Ellis Avenue, Chicago, IL 60637, USA

${ }^{13}$ Enrico Fermi Institute, University of Chicago, 5640 South Ellis Avenue, Chicago, IL 60637, USA 
${ }^{14}$ Department of Astronomy and Astrophysics, University of Chicago, 5640 South Ellis Avenue, Chicago, IL 60637, USA

${ }^{15}$ Dalhousie University, Halifax, Nova Scotia, Canada

${ }^{16}$ European Southern Observatory, Karl Schwarzschild Straße 2, 85748 Garching, Germany

${ }^{17}$ Department of Astronomy and Physics, Saint Mary's University, Halifax, Nova Scotia, Canada

${ }^{18}$ Department of Physics, University of California, One Shields Avenue, Davis, CA 95616, USA

${ }^{19}$ Department of Astronomy, University of Florida, Bryant Space Sciences Center, Gainesville, FL 32611 USA

${ }^{20}$ Department of Physics and Astronomy, University College London, Gower Street, London WC1E 6BT, UK

${ }^{21}$ Kavli Institute for Particle Astrophysics and Cosmology, Stanford University, Stanford, CA 94305, USA

${ }^{22}$ Department of Physics and Astronomy, McMaster University, Hamilton, ON L8S 4M1 Canada

${ }^{23}$ Department of Physics and Astronomy, University of California, Los Angeles, CA 90095-1547, USA

${ }^{24}$ National Radio Astronomy Observatory, 520 Edgemont Road, Charlottesville, VA 22903, USA

${ }^{25}$ Large Synoptic Survey Telescope, 950 North Cherry Avenue, Tucson, AZ 85719, USA

${ }^{26}$ Max-Planck-Institut für Radioastronomie, Auf dem Hügel 69 D-53121 Bonn, Germany

${ }^{27}$ International Max Planck Research School (IMPRS) for Astronomy and Astrophysics, Universities of Bonn and Cologne

${ }^{28}$ Hubble Fellow

According to the current understanding of cosmic structure formation, the precursors of the most massive structures in the Universe began to form shortly after the Big Bang, in regions corresponding to the largest fluctuations in the cosmic density field ${ }^{1+3}$. Observing these structures during their period of active growth and assembly - the first few hundred million years of the Universe-is challenging because it requires surveys that are sensitive enough to detect the distant galaxies that act as signposts for these structures and wide enough to capture the rarest objects. As a result, very few such objects have been detected so far 45 . Here we report observations of a far-infrared-luminous object at redshift 6.900 (less than $800 \mathrm{Myr}$ after the Big Bang) that was discovered in a wide-field survey ${ }^{6}$. High-resolution imaging reveals this source to be a pair of extremely massive star-forming galaxies. The larger of these galaxies is forming stars at a rate of 2900 solar masses per year, contains 270 billion solar masses of gas and 2.5 billion solar masses of dust, and is more massive than any other known object at a redshift of more than 6. Its rapid star formation is probably triggered by its companion galaxy at a projected separation of just 8 kiloparsecs. This merging companion hosts 35 
billion solar masses of stars and has a star-formation rate of 540 solar masses per year, but has an order of magnitude less gas and dust than its neighbor and physical conditions akin to those observed in lower-metallicity galaxies in the nearby Universe $\mathrm{e}^{7}$. These objects suggest the presence of a dark-matter halo with a mass of more than $\mathbf{4 0 0}$ billion solar masses, making it among the rarest dark-matter haloes that should exist in the Universe at this epoch.

SPT0311-58 (SPT-S J031132-5823.4) was originally identified in the $2500 \mathrm{deg}^{2}$ South Pole Telescope (SPT) survey ${ }^{89}$ as a luminous source (flux densities of 7.5 and $19.0 \mathrm{mJy}$ at wavelengths of $2.0 \mathrm{~mm}$ and $1.4 \mathrm{~mm}$ ) with a steeply rising spectrum, indicative of thermal dust emission. Observations with the Atacama Large Millimeter/submillimeter Array (ALMA) provide the redshift of the source. The $J=6-5$ and $7-6$ rotational transitions of the carbon monoxide molecule, along with the ${ }^{3} P_{2}-{ }^{3} P_{1}$ fine structure transition of atomic carbon, were found redshifted to $87-103 \mathrm{GHz}$ in a wide spectral scan ${ }^{6}$. The frequencies and spacings of these lines unambiguously place the galaxy at $z=6.900(2)$, corresponding to a cosmic age of $780 \mathrm{Myr}$ (using recent cosmological parameters $\frac{10}{10}$ of Hubble constant $H_{0}=67.7 \mathrm{~km} / \mathrm{s} / \mathrm{Mpc}$, matter density $\Omega_{\mathrm{m}}=0.309$, and vacuum energy density $\Omega_{\Lambda}=0.691$ ). An elongated faint object is seen at optical and near-infrared wavelengths, consistent with a nearly edge-on spiral galaxy at $z=1.4 \pm 0.4$ acting as a gravitational lens for the background source (see Methods section 'Modelling the SED'; here and elsewhere the error range quoted corresponds to a $1 \sigma$ uncertainty). Together these observations indicate that SPT0311-58 is the most distant known member of the population of massive, infrared-bright but optically dim dusty galaxies identified with ground- and space-based wide-field surveys 11 .

The far-infrared emission from SPT0311-58 provides an opportunity to study its structure with little confusion from the foreground galaxy. We conducted ALMA observations at $\sim 0.3^{\prime \prime}$ resolution at three different frequencies (see Methods), 240, 350, and $420 \mathrm{GHz}$, corresponding to rest-frame 160, 110, and $90 \mu \mathrm{m}$. The observations at $240 \mathrm{GHz}$ include the $158 \mu \mathrm{m}$ fine-structure line of ionized carbon ([C II $]$ ) and those at $420 \mathrm{GHz}$ the $88 \mu \mathrm{m}$ fine-structure line of doubly ionized oxygen $\left(\left[\mathrm{O}_{\mathrm{III}}\right]\right)$. The $160 \mu \mathrm{m}$ continuum and $\left[\mathrm{C}_{\mathrm{II}}\right]$ and $\left[\mathrm{O}_{\mathrm{III}}\right]$ line emission maps of the source are shown in Fig 1. Two emissive structures are visible in the map, SPT0311-58E and SPT0311$58 \mathrm{~W}$, which are separated by less than $2^{\prime \prime}$ on the sky, before correction for gravitational deflection. While the morphology of the eastern and western sources is reminiscent of a lensing arc (W) and counter-image $(\mathrm{E})$, the $\left[\mathrm{C}_{\mathrm{II}}\right]$ line clarifies the physical situation. SPT0311-58E is separated from the brighter $\mathrm{W}$ source by $700 \mathrm{~km} \mathrm{~s}^{-1}$ and is therefore a distinct galaxy.

Lens modeling of the 160,110 , and $90 \mu \mathrm{m}$ continuum emission from this source was performed using a pixellated reconstruction technique ${ }^{\sqrt{12}}$ (Fig. 16, Extended Data Fig. 5, and Methods, 
Section 'Gravitational lens modelling'). The source structure and lensing geometry is consistent between the observations, and indicates that the two galaxies are separated by a projected (proper) distance of $8 \mathrm{kpc}$ in the source plane. SPT0311-58E has an effective radius of $1.1 \mathrm{kpc}$, while SPT0311-58W shows a clumpy, elongated structure $7.5 \mathrm{kpc}$ across. The (flux-weighted) sourceaveraged magnifications of each galaxy and of the system as a whole are quite low $\left(\mu_{\mathrm{E}}=1.3\right.$, $\mu_{\mathrm{W}}=2.2, \mu_{\mathrm{tot}}=2.0$ ) because the $\mathrm{W}$ source is extended relative to the lensing caustic and the E source is far from the region of high magnification. The same lensing model applied to the channelized [ $\mathrm{C}$ II] data reveals a clear velocity gradient across SPT0311-58W which may be due to either rotational motions or a more complicated source structure coalescing at the end of a merger.

With the lensing geometry characterized, it is clear that the two galaxies that comprise SPT0311-58 are extremely luminous. Their intrinsic infrared $(8-1000 \mu \mathrm{m})$ luminosities have been determined from observations of rest-frame ultraviolet to submillimeter emission (see Methods, Section 7), and are $L_{\mathrm{IR}}=(4.6 \pm 1.2) \times 10^{12}$ and $(33 \pm 7) \times 10^{12} L_{\odot}$ for $\mathrm{E}$ and $\mathrm{W}$, respectively. Assuming that these sources are powered by star formation, as suggested by their extended far-infrared emission, these luminosities are unprecedented at $z>6$. The implied (magnificationcorrected) star formation rates are correspondingly enormous, $540 \pm 175$ and $2900 \pm 1800 M_{\odot} \mathrm{yr}^{-1}$, likely due to the increased instability associated with the tidal forces experienced by merging galaxies ${ }^{13}$. The components of SPT0311-58 have luminosities and star formation rates similar to the other massive $z>6$ galaxies identified by their dust emission, including HFLS3 $(z=6.34)$, which has a star formation rate of $1300 M_{\odot} \mathrm{yr}^{-1}$ after correcting for a magnification factor ${ }^{14}$ of 2.2 , and a close quasar-galaxy pair resolved recently with $\mathrm{ALMA}^{15}$ at $z=6.59$, which are forming stars at rates of 1900 and $800 M_{\odot} \mathrm{yr}^{-1}$. Unlike the latter case, however, there is no evidence of a black hole in either source in SPT0311-58.

Unlike any other massive dusty source at $z>6$, the rest-frame ultraviolet emission of SPT0311-58E is clearly detectable with modest integration by the Hubble Space Telescope. The detected ultraviolet luminosity $\left(L_{\mathrm{UV}}=7.4 \pm 0.7 \times 10^{10} L_{\odot}\right)$ suggests a star formation rate of just $13 M_{\odot} \mathrm{yr}^{-1}, 2 \%$ of the rate derived from the far-infrared emission, which is consistent with SPT0311-58E forming most of its stars behind an obscuring veil of dust. The inferred stellar mass for this galaxy (see Methods Sec. 7) is $(3.5 \pm 1.5) \times 10^{10} M_{\odot}$. While no stellar light is convincingly seen from SPT0311-58W, the absence of rest-frame ultraviolet emission is likely explained by heavy dust obscuration and is not unusual ${ }^{16}$. Although SPT0311-58E is the less massive of the two components, even this galaxy is rare among ultraviolet-detected galaxies at $z \sim 7$. Such galaxies are found in blank field surveys to have a sky density of just 1 per 30 square arcminutes 17 . 
The far-infrared continuum and line emission of SPT0311-58E and W seen in Fig. 1(d-f) imply substantial differences in the physical conditions in these objects. Compared to the western source, SPT0311-58E has a higher ratio of [C II] line to $160 \mu \mathrm{m}$ continuum and a much larger luminosity ratio between $[\mathrm{O} \mathrm{III}]$ and $[\mathrm{C} \mathrm{II}]$. The $[\mathrm{O} \mathrm{III}]$ emission is dramatically more luminous in the eastern source, with most of the western source (excluding the southern end) showing no emission at all. Because the formation of $\mathrm{O}^{++}$ions requires photons with energies of more than $35.1 \mathrm{eV}$, this line arises only in ionized regions around the hottest stars or near active galactic nuclei ${ }^{18}$. It is unlikely that active galactic nuclei are the origin for the [O III] line in SPT0311-58E, because both the continuum and line emission extend across most of the galaxy rather than being concentrated in a putative nuclear region. Observations of [O $\mathrm{III}] 88 \mu \mathrm{m}$ emission in actively star forming galaxies at low ${ }^{719}$ and high ${ }^{20}$ redshift have found that the line luminosity ratio between [O III] and [C II] rises as gas metallicity decreases. The ultraviolet photons capable of forming $\mathrm{O}^{++}$have a longer mean free path in a lower-metallicity interstellar medium than in a higher-metallicity one, and the electron temperature remains higher for the same ionizing flux, both of which favor increased [O III] emission ${ }^{21}$. The difference in the $\left[\mathrm{C}_{\mathrm{II}}\right]$ line-to-continuum ratio may result from multiple effects: the known suppression ${ }^{22}+24$ of the [C II] to- $L_{\mathrm{IR}}$ ratio in regions of increased star formation surface density (higher in SPT0311-58W), and the increased [C II] to- $L_{\mathrm{IR}}$ ratio in star-forming galaxies of lower metallicity ${ }^{7}$. Whether SPT0311-58E (or the southern edge of SPT0311-58W, which is similar to $\mathrm{E}$ in these properties) has a more primordial interstellar medium than the bulk of SPT0311-58W can be tested in future observations.

The masses of the components of SPT0311-58 are remarkable for a point just 780 Myr after the Big Bang. Fig. 2 compares SPT0311-58 to objects at $z>5$ for which we have estimates of dust mass $\left(M_{\text {dust }}\right)$ and/or total gas mass $\left(M_{\text {gas }}\right)$. For SPT0311-58 the best constraints on both of these quantities come from the joint analysis ${ }^{\sqrt{6}}$ of its far-infrared continuum and line emission, specifically the rotational transitions of carbon monoxide and neutral carbon. Here we have divided these masses among the two galaxies according to the lensing-corrected ratio of dust continuum emission (6.7) observed in our three high-resolution ALMA continuum observations, as the dust continuum luminosity is roughly proportional to the dust mass. The corresponding dust and gas masses are $M_{\text {gas }}=(2.7 \pm 1.7) \times 10^{11}(\mathrm{~W})$ and $(0.4 \pm 0.2) \times 10^{11} M_{\odot}(\mathrm{E})$, and $M_{\text {dust }}=(2.5 \pm 1.6) \times 10^{9}$ (W) and $(0.4 \pm 0.2) \times 10^{9} M_{\odot}(\mathrm{E})$. The gas mass can also be estimated using the CO luminosity, though the conversion between luminosity and gas mass in this optically thick line is well-known to vary substantially depending on many factors, including star formation intensity and metallicity ${ }^{25}$. Taking the observed ${ }^{6}$ luminosity in the $J=3-2$ line of $\mathrm{CO}$, converting to $J=1-0$ under the conservative assumption of thermalized emission, and connecting luminosity to mass with a 
standard value of $\alpha_{\mathrm{CO}}=1.0 M_{\odot}\left(\mathrm{K} \mathrm{km} \mathrm{s}^{-1} \mathrm{pc}^{2}\right)^{-1}$, we derive $M_{\text {gas }}=(6.6 \pm 1.7) \times 10^{10}(\mathrm{~W})$ and $M_{\text {gas }}=(1.0 \pm 0.3) \times 10^{10} M_{\odot}(\mathrm{E})$. SPT0311-58W stands out above all of the known galaxies at $z>6$, that is, during the first $\sim 900$ Myr of cosmic history.

SPT0311-58 highlights an early and extreme peak in the cosmic density field and presents an opportunity to test the predictions for the growth of structure in the current cosmological model. The mass of the dark matter halo that hosts this system is uncertain, but it can be estimated in several ways. Considering the gas masses of the two galaxies, which, for most massive star forming galaxies ${ }^{2627}$, represent the dominant component of baryons that have cooled and assembled at the center of the dark matter halo, the cosmic baryon fraction ${ }^{10} f_{\mathrm{b}}=0.19$ places a hard lower bound on the total halo mass of $4 \times 10^{11} M_{\odot}$ for the lower ( $\alpha_{\mathrm{CO}}$-based) gas mass estimate. A less conservative assumption incorporates the knowledge, based on observations across a wide range of redshifts, that only a fraction of the baryons in a dark-matter halo (less than one-quarter, $M_{\mathrm{b}} / M_{\text {halo }}=0.05$; see figure 15 of Ref. 3 ) are destined to accrue to the stellar mass of the central galaxy ${ }^{3}$. In this case, a total halo mass of $(1.4-7.0) \times 10^{12} M_{\odot}$ is implied, depending on which estimate of gas mass is adopted. To understand the rareness of the dark matter halo hosting SPT0311-58, we calculate curves describing the rarest halos that should exist in the universe at any redshift ${ }^{28}$. In Fig. 3 we show the halo masses inferred for many high-redshift galaxies, using the same path of gas mass to halo mass described above, finding that SPT0311-58 is indeed closest to the exclusion curves and therefore marks an exceptional peak in the cosmic density field at this time.

We have found a system of massive, rapidly star forming, dusty galaxies at $z=6.900$, the most distant galaxies of this type yet discovered. Two compact and infrared-luminous galaxies are seen separated by less than $8 \mathrm{kpc}$ in projection, and $700 \mathrm{~km} \mathrm{~s}^{-1}$ in velocity, probably in the process of forming one of the most massive galaxies of the era. Even before coalescence, the larger galaxy in the pair is more massive than any other galaxy known at $z>6$. Although the discovery of such a system at this high redshift and in a survey that covered less than $10 \%$ of the sky is unprecedented, its existence is not precluded by the current cosmological paradigm. 

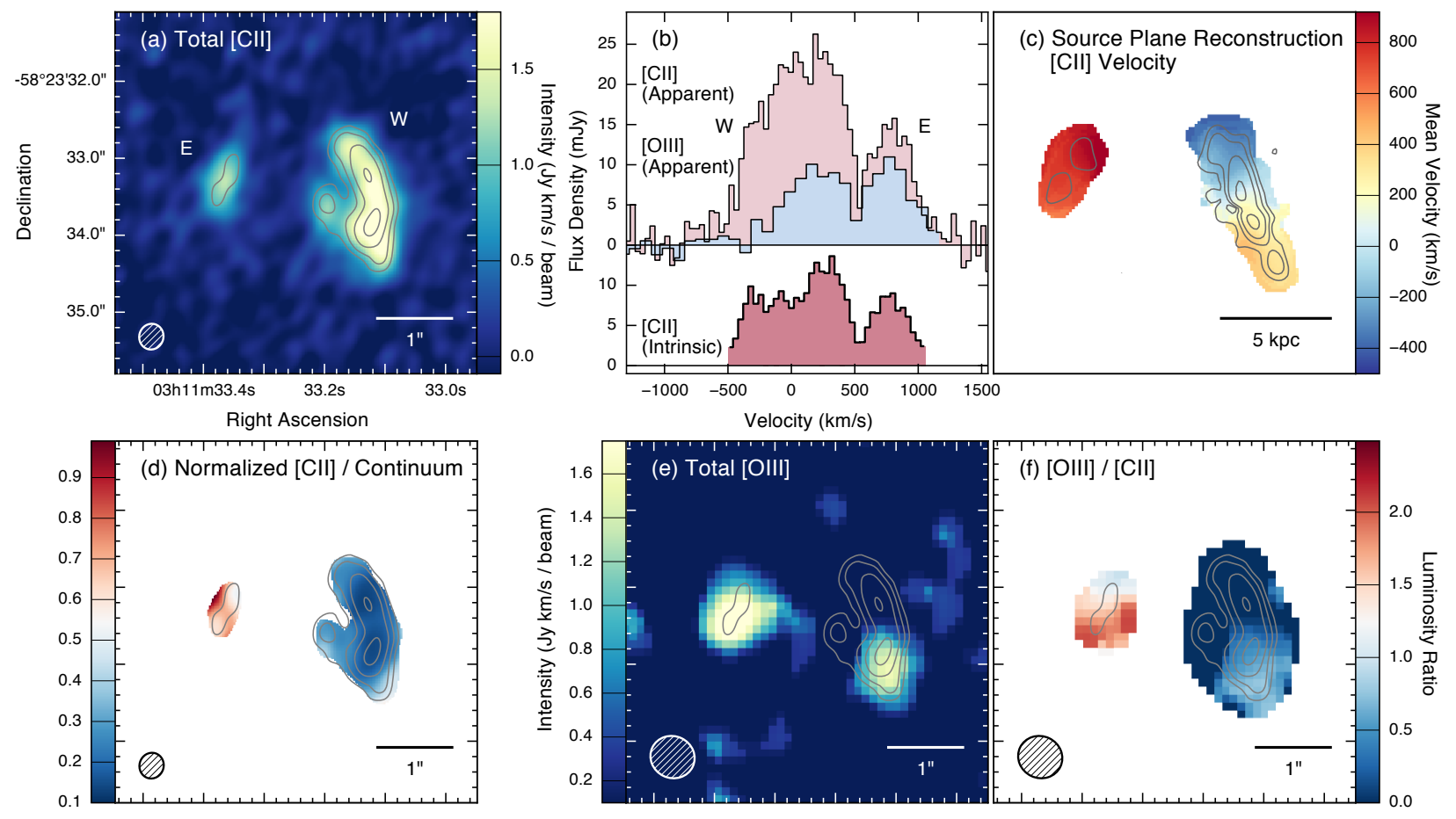

Figure 1 | Continuum, [C II], and [O III] emission from SPT0311-58 and the inferred source-plane structure. (a) Emission in the $157.74 \mu \mathrm{m}$ fine structure line of ionized carbon ([C II]) as measured at $240.57 \mathrm{GHz}$ with ALMA, integrated across $1500 \mathrm{~km} \mathrm{~s}^{-1}$ of velocity, is shown with the color scale. The range in flux per synthesized beam (the $0.25 \times 0.30^{\prime \prime}$ beam is shown in the lower left), is provided at right. The restframe $160 \mu \mathrm{m}$ continuum emission, measured simultaneously, is overlaid with contours at $8,16,32,64$ times the noise level of $34 \mu \mathrm{Jy}_{\text {beam }^{-1}}$. SPT0311-58E and $\mathrm{W}$ are labeled. (b) The continuum-subtracted, sourceintegrated $[\mathrm{C} \mathrm{II}]$ and $[\mathrm{O} \mathrm{III}]$ spectra. The upper spectra are as observed ("apparent") with no correction for lensing, while the lensing-corrected ("intrinsic") [C II] spectrum is shown at bottom. The E and W sources separate almost completely at a velocity of $500 \mathrm{~km} \mathrm{~s}^{-1}$. (c) The source-plane structure after removing the effect of gravitational lensing. The image is colored by the flux-weighted mean velocity, showing clearly that the two objects are physically associated but separated by roughly $700 \mathrm{~km} \mathrm{~s}^{-1}$ in velocity and $8 \mathrm{kpc}$ (projected) in space. The reconstructed $160 \mu \mathrm{m}$ continuum emission is shown in contours. A scale bar in the lower right represents the angular size of $5 \mathrm{kpc}$ in the source plane. (d) The line-to-continuum ratio at the $158 \mu \mathrm{m}$ wavelength of [C II], normalized to the map peak. The [C II] emission from SPT0311-58E is significantly brighter relative to its continuum than for $\mathrm{W}$. The sky coordinates and rest-frame $160 \mu \mathrm{m}$ continuum contours of Fig. 1/d), (e), and (f) are the same as in panel (a). (e) Velocity-integrated emission in the $88.36 \mu \mathrm{m}$ fine structure line of doubly-ionized oxygen ([O III]) as measured at $429.49 \mathrm{GHz}$ with ALMA. The data have an intrinsic angular resolution of $0.2 \times 0.3^{\prime \prime}$ but have been tapered to $0.5^{\prime \prime}$ owing to the lower signal-to-noise in these data. (f) The luminosity ratio between the [O III] and [C II] lines. As in the case of the $[\mathrm{C}$ II] line-to-continuum ratio, a significant disparity is seen between the E and W galaxies of SPT0311-58. 


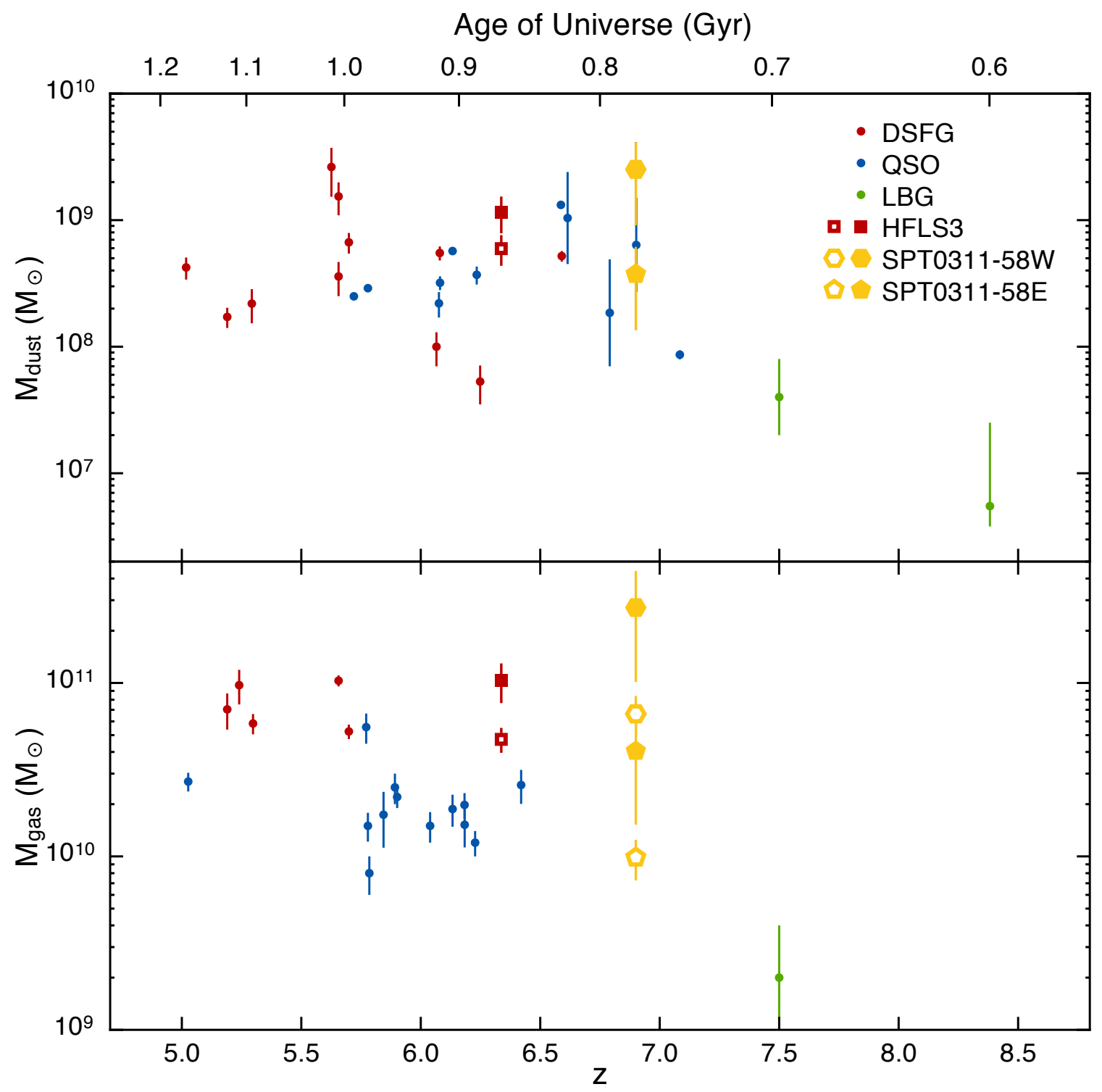

Figure 2 | Mass measurements for high-redshift galaxies. Dust masses are taken from the literature, as described in the Methods. Gas masses are primarily derived from observations of various CO rotational transitions, with the literature line luminosities converted to molecular gas masses under standardized assumptions (see Methods). The comparison sample is divided among three object classes: DSFGs, quasars (QSO), and Lyman-break galaxies (LBG). These objects are typically selected by FIR emission (DSFGs) or optical/infrared emission (QSOs, LBGs). Three additional DSFGs, SPT0311-58E (yellow pentagons), SPT0311-58W (yellow hexagons) and HFLS3 (red squares), have extensive photometry and line measurements, which enable more sophisticated estimates of their dust and gas masses $\frac{629}{2}$ from a combined analysis of the dust and CO line emission. For these objects we also show masses derived under the simpler analysis as open symbols (for SPT0311-58 the methods give very similar answers for $M_{\text {dust }}$ ). Error bars represent $1 \sigma$ uncertainties. 


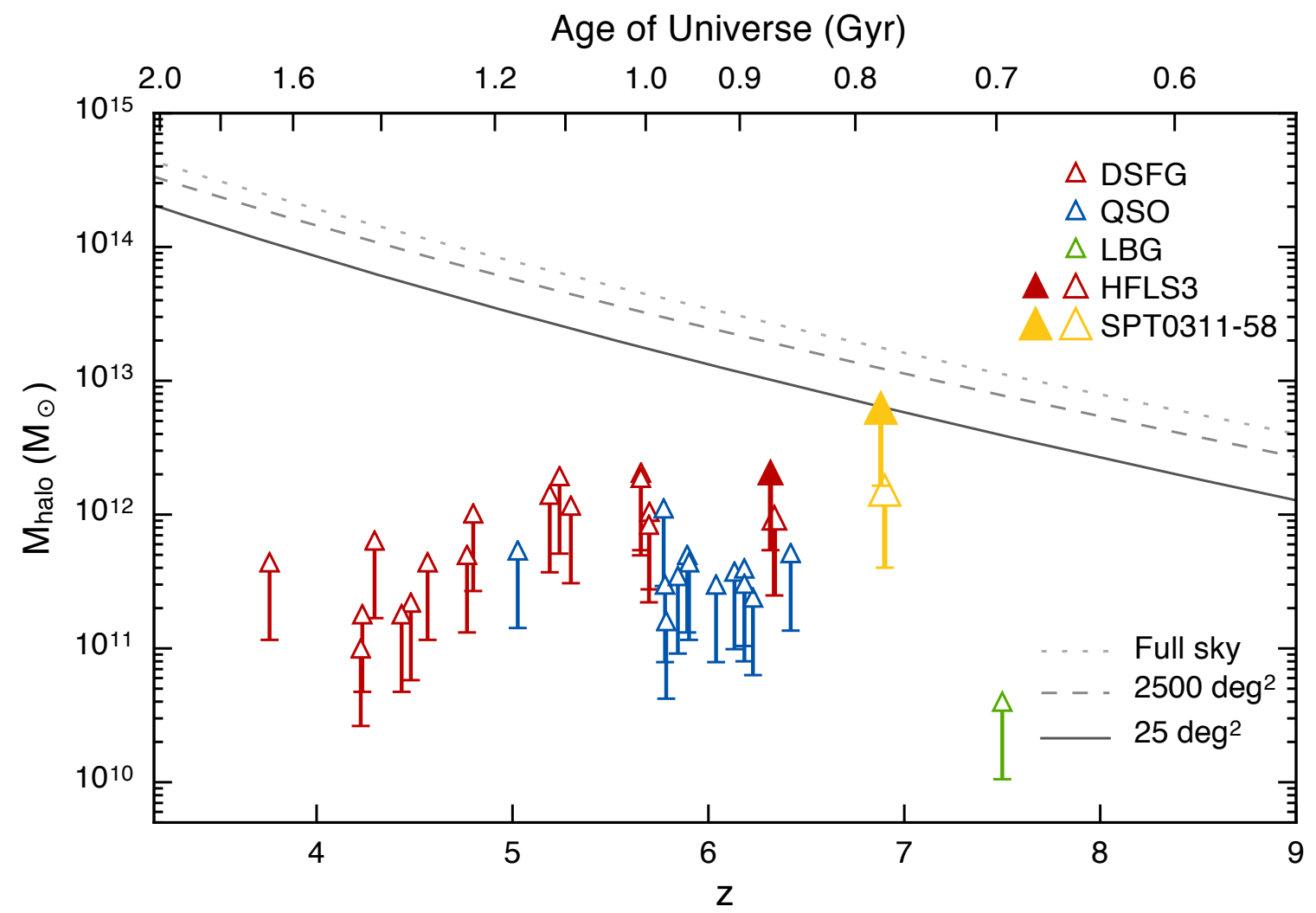

Figure 3 Halo masses for rare, high-redshift massive galaxies. The dark matter halo mass ( $M_{\text {halo }}$; defined at an overdensity of 200 times the mean matter density of the universe) is inferred for galaxies in the first 2 Gyr after the Big Bang (see Methods). These masses present a range of lower limits, from the most conservative assumption (lower bars) that all baryons in the initial halo have been accounted for in the molecular gas mass, to the observationally motivated assumption (upper triangles) that the baryonic mass $\left(M_{\mathrm{b}}\right)$ in gas is a fixed ratio of the halo mass $M_{\mathrm{b}} / M_{\text {halo }}=0.05$, calibrated through a comparison ${ }^{3}$ of simulations and observations spanning $z=0-8$. The most massive halos that are expected to be observable ${ }^{\sqrt{28}}$ within the whole sky (dotted line), the $2500 \mathrm{deg}^{2}$ area of the SPT survey (dashed line), and within the subset of that area that is magnified by a factor of two or more (solid line) are also plotted as a function of redshift. As SPT0311-58E and W reside within the same halo, they are combined for this analysis. As in Fig. 2, halo masses are derived for HFLS3 (large red triangles) and SPT0311-58 (large yellow triangles) using only the CO luminosity (open symbols) and the more sophisticated dust and $\mathrm{CO}$ analysis (filled symbols); the pairs of points are slightly offset in redshift for clarity. 


\section{Methods}

\section{ALMA Millimeter/Submillimeter Interferometry}

We have acquired four observations of SPT0311-58 with ALMA in four receiver bands (B3, B6, B7, B8, covering 84 to $432 \mathrm{GHz}$ ) under projects 2015.1.00504.S (PI: Strandet) and 2016.1.01293.S (PI: Marrone). A summary of these observations, including dates, calibration sources, integration times, atmospheric opacity, noise levels, and resolution, is provided in Extended Data Table 1. Salient details are provided below for each observation.

The redshift of SPT0311-58 and the $3 \mathrm{~mm}$ continuum flux density were determined from an 84.2-114.9 GHz spectrum assembled from five separate tunings in ALMA band 3 under ALMA Cycle 3 project 2015.1.00504.S. The observing strategy has been used to discover the redshifts of more than 50 SPT dusty sources, and further details on the redshift coverage are provided in previous works 30 31. Data were taken on 2015 December 28 and 2016 January 2 in ALMA configuration C36-1 (baseline lengths of 15-310 meters) using 34 and 41 antennas, respectively. The resulting image has a resolution of $3.3 \times 3.5^{\prime \prime}$, though there is spatial information on somewhat finer scales that allows us to separately estimate flux densities for the E and $\mathrm{W}$ sources, separated by $\sim 2^{\prime \prime}$. Further details of the analysis are provided by Strandet et al. ${ }^{6}$.

ALMA observed SPT0311-58 a second time under 2015.1.00504.S in band 7 (LO = 343.48 $\mathrm{GHz}$ ) to produce a continuum image suitable for gravitational lens modeling. Similar observations were used to produce lens models of SPT sources in previous cycles ${ }^{24 / 32}$. The observations were performed with 41 antennas in the C40-4 configuration, providing 15-770 meter baselines. The resulting image has an angular resolution of $0.3 \times 0.5^{\prime \prime}$, though lacking any spectral lines it was found to be insufficient to provide an unambiguous determination of the lensing configuration.

The ALMA Cycle 4 project 2016.1.01293.S was intended to follow up on the discovery of this very distant source through spectroscopic observations. The $158 \mu \mathrm{m}$ line of [C $\mathrm{CI}$ ] was observed on 2016 November 3 in ALMA configuration C40-5, which provided baseline lengths of 18-1120 meters. This provides the primary imaging for this work, as it yielded an extremely significant detection of the $\left[\mathrm{C}_{\mathrm{II}}\right]$ line and continuum structure at high resolution.

A final observation was obtained in ALMA band $8(\mathrm{LO}=423.63 \mathrm{GHz})$, in configuration C404 (baselines 15-920 meters). The observations were repeated in four segments to yield the required 
integration time. The resulting data have $0.2 \times 0.3^{\prime \prime}$ resolution. These data provide a final spatiallyresolved continuum observation, at $90 \mu \mathrm{m}$ rest-frame wavelength, along with spectroscopic images of the $88 \mu \mathrm{m}$ line of [O III]. The ALMA continuum images are shown in Extended Data Fig. 1 .

\section{Spitzer Infrared Imaging}

Infrared observations of SPT0311-58 were acquired with the Infrared Array Camera (IRAC) instrument ${ }^{33}$ on the Spitzer Space Telescope as a part of Cycle 24 Hubble Space Telescope program 14740 (PI: Marrone). The observations consisted of 95 dithered 100 s exposures on-source in both operable IRAC arrays at 3.6 and $4.5 \mu \mathrm{m}$. A large dither throw was used. The dataset thus had sufficiently high redundancy to support our standard reduction procedure, which involved constructing an object-masked median stack of all 95 exposures in each band, and then subtracting the median stack from the raw frames to compensate for bad pixels not automatically masked by the pipeline, and remove gradients in the background. After these initial preparatory steps, the background-subtracted exposures were combined in the standard way ${ }^{34}$ with IRACproc ${ }^{35}$ and MOPEX to create mosaics having $0.6^{\prime \prime}$ pixels. The mosaics achieved an effective total integration time of about $9000 \mathrm{~s}$ after masking cosmic rays and other artifacts. Two flanking fields were covered to the same depth but separately, each in one IRAC passband.

Photometry was performed on the mosaics using Source Extractor ${ }^{\sqrt{36}}$ (SE) in dual-image mode after trimming to exclude the flanking fields and unexposed areas. The lens galaxy associated with SPT0311-58 was well detected with no evidence for saturation or even nonlinear detector behavior. During this process background and object images were generated and inspected to verify that SE performed as expected and generated valid photometry.

\section{Hubble Space Telescope Imaging}

SPT0311-58 was observed for 5 orbits of HST imaging with ACS and WFC3/IR in Cycle 24 (PID 14740; PI: Marrone) to determine the morphology of the foreground lens and better constrain the spectral energy distribution of both the lens and source. All observations were acquired on 30 April 2017. The ACS imaging consists of a single orbit divided between the F606W and F775W filters. Exposure times are $844 \mathrm{~s}$ and $1.5 \mathrm{ks}$, respectively. Four orbits of WFC3/IR observing was split evenly between the F125W and F160W filters. While the nominal exposure times are $5.6 \mathrm{ks}$, a subset of the data in both filters was compromised by significant contamination from scattered 
earthlight. We reprocessed the imaging to remove contaminated data, resulting in final exposure times of $4.9 \mathrm{ks}$ in each band.

\section{Gemini Optical/IR Imaging and Spectroscopy}

With the Gemini Multi-Object Spectrograph ${ }^{37}$ (GMOS) of Gemini-South, we obtained deep $i$ and $z$ images of SPT0311-58 (Program: GS-2015B-Q-51, PI: Rotermund) on 2016 January 29 and 31. The instrument consists of three 2048 x 4176 pixel CCDs, separated by two $6.46^{\prime \prime}$ (80 pixel) gaps, with a scale of $0.0807^{\prime \prime}$ pixel $^{-1}$. The field of view (FOV) of the GMOS camera is $5.5^{\prime} \times 5.5^{\prime}$. Our images were taken under photometric conditions and using a $2 \times 2$ binning, which gives a scale of $0.161^{\prime \prime}$ pixel $^{-1}$. The total integration times were $3600 \mathrm{~s}$ for $i$-band and $6600 \mathrm{~s}$ for $z$-band, with average seeing conditions of $1.3^{\prime \prime}$ and $1.0^{\prime \prime}$ in $i$-band and $z$-band, respectively. The resulting $5 \sigma$ point source depths were $i_{A B}=25.2$ and $z_{A B}=25.0$.

SPT0311-58 was observed using the Facility Near-Infrared Wide-field Imager \& MultiObject Spectrograph for Gemini (FLAMINGOS-2) ${ }^{\sqrt[38]{3}}$ at the Gemini-South Observatory on the nights of UT 2016 September 23, and 2017 February 06, under Program GS-2016B-Q-68. The instrument was used in imaging mode, with $0.181^{\prime \prime}$ pixels, and yielding an unvignetted circular field of view of $\sim 5.5^{\prime}$ diameter. Our observing sequence for the survey consisted of a randomly ordered dither pattern, with $15^{\prime \prime}$ offsets about the pointing center. This pattern was repeated until the required total exposure time was achieved. The individual $\mathrm{K}_{s}$-band exposure time was set at $15 \mathrm{~s}$ in the first observation, and $10 \mathrm{~s}$ in the second observation, yielding a typical background sky level in $K_{s}$ of $\sim 10,000-12,000$ counts (detector nonlinearity can be corrected to better than $1 \%$ up to 45,000 counts), ensuring that 2MASS stars with $K_{s}>13$ do not saturate and can be used for photometric calibration. The data were reduced using the python-based FLAMINGOS-2 Data Pipeline, FATBOY ${ }^{39}$, created at the University of Florida. Briefly, a calibration dark was subtracted from the data set, a flat field image and a bad pixel map were created, and the flat field was divided through the data. Sky subtraction was performed to remove small-scale structure with a subsequent low order correction for the large-scale structure. Finally, the data were aligned and stacked. The seeing conditions averaged $0.7^{\prime \prime}$ in the final image comprising 44 minutes of integration, reaching $K_{s, A B}=23.6$ at $5 \sigma$.

Spectroscopy was obtained with the GMOS-S instrument on the nights of UT 2016 February 1 and 2 (Program: GS-2016B-Q-68, PI: Chapman) using the $1^{\prime \prime}$ wide longslit at a position angle = $-10^{\circ}$ east of north, and the instrument configured with the R400 grating and $2 \times 2$ detector binning. 
For a source that fills the $1^{\prime \prime}$ slit this setup results in a spectral resolution of $\sim 7 \AA$. The observations were spectrally dithered, using two central wavelength settings ( 8300 and $8400 \AA)$ to cover the chip gaps. The data comprise a series of individual 900s exposures, dithering the source spatially between two positions ("A" and "B") along the slit in an ABBA pattern, repeated four times, two at each central wavelength setting. The total integration time is $4 \mathrm{hrs}$. A bright foreground object was positioned along the slit midway between the acquisition star and SPT0311-58, providing an additional reference point for locating traces along the slit.

The spectra were reduced, beginning with bias subtraction and bad pixel masking using the IRAF GMOS package provided by Gemini. The individual chips were combined into a single mosaic for each exposure and the mosaicked frames were then sky subtracted by differencing neighboring A-B exposure pairs; this method resulted in nearly Poisson noise even under the numerous bright sky lines. A flat-field slit illumination correction was applied and a wavelength calibration derived for each mosaic. The 2-dimensional spectrum was created by median combining the individual exposure frames.

The spectrum shows a faint continuum beginning above $9000 \AA$ at the location of SPT031158. A one-dimensional extraction of the faint trace yields no reliable redshift measurement, but it is consistent with the redshifted $4000 \AA$ break that is expected for the foreground galaxy at $z \sim 1.4$. Calibrated against the nearby $R=16.4$ star spectrum we find no flux at the expected location of Ly $\alpha$ redshifted to $z=6.900(\sim 9600 \AA)$ ) down to a $3 \sigma$ flux limit of $3.0 \times 10^{-17} \mathrm{erg} \mathrm{s}^{-1} \mathrm{~cm}^{-2}$ for a $500 \mathrm{~km} \mathrm{~s}^{-1}$ wide emission line.

\section{Image de-blending}

At the position of SPT0311-58, our optical and infrared images (Extended Data Fig. 2) show a prominent lower-redshift galaxy responsible for lensing the western source, as well as direct stellar emission from the eastern source in the Hubble images (Extended Data Fig. 3), which have the highest resolution. To extract reliable photometry for SPT0311-58E, particularly in the lowresolution Spitzer images that cover the rest-frame optical, and to search for emission from $\mathrm{W}$ underneath the lens galaxy, we must model and remove the lens emission. We follow procedures similar to those in past work ${ }^{41}$, using the HST/WFC3 images as the source of the lens galaxy model to de-blend the IRAC image. The foreground lens can be fit with a single Sérsic profile with an index $n=1.77$. As seen in Extended Data Fig. 4, there is no clear rest-frame ultraviolet emission from SPT0311-58W in the HST bands after removal of the lens model. To remove the lens from 
the IRAC image, the WFC3 model is convolved with the IRAC point spread function and then subtracted from the 3.6 and $4.5 \mu \mathrm{m}$ images. Residual emission is seen near the positions of both the eastern and western sources. Unfortunately, because SPT0311-58W lies right on top of the lens, the residuals are extremely susceptible to image deconvolution errors and we do not believe the Spitzer/IRAC fluxes to be reliable. In contrast SPT0311-58E is one full IRAC resolution element, $1.7^{\prime \prime}$, from the lens centroid, and we consider the residual emission at this position to be usable in our subsequent analyses. Images of the model and residuals are provided in Extended Data Fig. 4 and the resulting photometry is provided in Extended Data Table 2.

\section{Gravitational Lens Modeling}

Gravitational lens modeling of SPT0311-58 was performed using two different codes that model the source-plane emission in different ways. Both codes fit directly to the visibilities measured by ALMA or other interferometers to avoid the correlated noise between pixels in inverted images. In each, the lens galaxy is modeled as a singular isothermal ellipsoid, and posterior parameter distributions are sampled using a Markov Chain Monte Carlo technique, marginalizing over several sources of residual calibration uncertainty (e.g., antenna-based phase errors).

Initial lens models were created using the visilens code, which is described in detail elsewhere ${ }^{24}$. The source plane is modeled as one or more elliptical Sérsic profiles. Because of the simplicity of this source-plane representation, the code is able to sample large and complex parameter spaces quickly. The continuum emission at 160,110, and $90 \mu \mathrm{m}$ was modeled with four Sérsic components, one for the SPT0311-58E and three for W. These models leave $\sim 8 \sigma$ peak residuals in the 160 and $90 \mu \mathrm{m}$ data, which both reach peak signal-to-noise $>150$.

After determining the lens parameters using visilens, we used the best-fit values as initial input to a pixellated reconstruction code ${ }^{12}$. This code represents the source plane as an array of pixels, rather than an analytic model, and determines the most probable pixel intensity values for each trial lens model while imposing a gradient-type regularization ${ }^{42}$ to avoid over-fitting the data. For each dataset, we fit for the strength of this regularization. At 160 and $90 \mu \mathrm{m}$ we re-fit for the lens model parameters and compare to the visilens models as a test of the robustness of the lens modeling. Within each code, the best-fit lens parameters at the two independent wavelengths are consistent to $<10 \%$. Further, both the lens parameters and source structure are consistent between the two independent codes, with intrinsic source flux densities, sizes, and magnifications that agree to $<15 \%$. The increased freedom in the source plane afforded by the pixellated reconstruction 
means that the lens parameters are not independently well-constrained by the $110 \mu \mathrm{m}$ data, which have both lower signal-to-noise and spatial resolution. For these data, we simply apply the lensing deflections determined from the other two datasets to reconstruct the source-plane emission. The pixellated reconstructions of the three continuum wavelengths are shown in Extended Data Fig. 5 .

The channelized [ $\left.\mathrm{C}_{\mathrm{II}}\right]$ line is also modeled using the same pixellated reconstruction technique, using 39 consecutive channels of $40 \mathrm{~km} / \mathrm{s}$ width, each with a peak signal-to-noise ranging from 9-34. For each channel, we apply the lensing deflections from the best-fit model of the $160 \mu \mathrm{m}$ data, which were observed simultaneously. We fit for the strength of the source-plane regularization ${ }^{12 / 42}$ at each channel, which varies across the line profile as some velocities experience higher magnification (e.g., those multiply imaged from $-280 \mathrm{~km} / \mathrm{s}$ to $+80 \mathrm{~km} / \mathrm{s}$ ) than others (e.g., the entire eastern source at $>+560 \mathrm{~km} / \mathrm{s}$ ). The models of each [C II] channel are represented in Extended Data Fig. 6 .

We determine the source magnifications using the $90 \mu \mathrm{m}$ pixellated model, in which the $\mathrm{E}$ source is detected at the highest signal-to-noise ratio and so the effects of varying the aperture used to measure the intrinsic flux density are minimized. Because the source-plane morphology is very similar between the three continuum wavelengths, the magnification is also essentially identical between them. We find flux-weighted, source-averaged magnifications for the eastern, western, and the system as a whole of $\mu_{\mathrm{E}}=1.3, \mu_{\mathrm{W}}=2.2, \mu_{\mathrm{tot}}=2.0$, respectively. These magnifications are substantially lower than the median magnification of 5.5 within the sample of 47 SPT-discovered dusty galaxies ${ }^{24}$ for which we have data adequate to construct lens models or conclude that sources are unlensed. In this case the low magnification is a consequence of the low mass of the lensing halo, typically expressed as an "Einstein" radius, $\theta_{E}$. The lens model for this source indicates $\theta_{E}=0.29^{\prime \prime}$, which is around the $10^{\text {th }}$ percentile for SPT lensed sources ${ }^{24}$, and the background source is both much larger than and offset from the regions of highest magnification. A large portion of the source is therefore only weakly magnified and the source-averaged values are low.

Finally, we also construct a lens model of the $95 \mathrm{GHz}$ ALMA data (rest-frame $380 \mu \mathrm{m}$; Extended Data Table 1). Because the spatial resolution of these data is low $\left(3.5^{\prime \prime}\right)$, we model these data using only the visilens code, which is more suited for low-resolution data. We only allow the lens parameters and source structural parameters (e.g., position, radius) to vary within the ranges determined from the higher-resolution 160, 110, and $90 \mu \mathrm{m}$ continuum data, leaving only the flux densities of the eastern and western objects as free parameters. This modeling indicates 
that essentially all of the observed $380 \mu \mathrm{m}$ emission can be ascribed to the western source, with the eastern source "detected" at $\sim 1 \sigma$.

In addition to the ALMA data, we use Herschel photometry reported previously $\sqrt{6}$ to constrain the SED of SPT0311-58E and W to rest-frame $30 \mu \mathrm{m}$ (250 $\mu \mathrm{m}$ observed). The resolution of Herschel SPIRE is not adequate to separate the two components, so we divide the total flux density observed in the three SPIRE bands between $\mathrm{E}$ and $\mathrm{W}$ according to the ratios observed in the ALMA bands. These photometric points are then corrected for the continuum magnification derived from the ALMA data and used in the SED modeling described below. The total and intrinsic flux densities are reported in Extended Data Table 3

\section{Modeling the Spectral Energy Distribution}

Extended Data Fig. 7 presents the spectral energy distributions of SPT0311-58E, W, and the foreground lens galaxy.

A photometric redshift for the lens is calculated with $E A Z Y^{43}$ using the data in Extended Data Table 2. The resulting redshift is 1.43 , with a $1 \sigma$ confidence interval of $1.08-1.85$. The lens SED fitting is performed with the Code Investigating GALaxy Emission (CIGALE ${ }^{44}$ 45) assuming $z=1.43$.

The multiple rest-frame ultraviolet to rest-frame optical detections of SPT0311-58E allow us to constrain the stellar mass using reasonable assumptions about the star formation history at this early point in cosmic history. The SED is fit by varying the e-folding time and age from a previously reported stellar population mode ${ }^{46}$ under single- and two-component formation histories, assuming solar metallicity and using a previously reported ${ }^{47}$ initial mass function. The minimum radiation field, power-law slope, and gamma, the fraction of dust mass exposed to radiation intensities above the minimum, from one dust mode ${ }^{48}$, and the color excess and attenuation slope from other dust models ${ }^{49}[50$ are kept free in the SED fitting. The AGN contribution is set to zero as there are no photometric points to constrain the spectral range most affected by AGN power (mid-infrared) and thus any fraction between 0 and $60 \%$ of the dust luminosity is attributable to AGN with nearly equal probability. However, this ignores the spatial distributions of the dust and line emission, which are not strongly peaked as is usually observed in AGN-dominated galaxies, so we deem this wide range to be unphysical. The inferred stellar mass and star formation rates are $(3.5 \pm 1.5) \times 10^{10} M_{\odot}$ and $540 \pm 175 M_{\odot} \mathrm{yr}^{-1}$, respectively, for the two-component star formation 
history. These answers agree within the uncertainties for a single-component star formation history. The infrared luminosity $\left(L_{\mathrm{IR}}\right.$, integrated from $\left.8-1000 \mu \mathrm{m}\right)$ is $(4.6 \pm 1.2) \times 10^{12} L_{\odot}$ and the extinction is $A_{V}=2.7 \pm 0.2 \mathrm{mag}$.

For the western source, we have only upper limits and the potentially-contaminated IRAC detections to constrain the rest-frame optical/ultraviolet emission. Accordingly, we use the IRAC photometry as upper limits, along with the HST limits and far-infrared data in Extended Data Table 3, and model the SED with CIGALE. We find a luminosity of $L_{\mathrm{IR}}=(33 \pm 7) \times 10^{12} L_{\odot}$, seven times larger than for the eastern source. A consistent luminosity is obtained by fitting the FIR SED with a modified blackbody 51 . The inferred star formation rate, which is closely connected to $L_{\mathrm{IR}}$, is $2900 \pm 1800 M_{\odot} \mathrm{yr}^{-1}$. Just as for the eastern source, the SED allows the AGN fraction to fall between 0 and $60 \%$ at roughly equal probability, so we take the absence of a dominant IR emission region (see Fig.s 11(c) and 5) as an indication that the AGN contribution is unlikely to be important and fix the AGN fraction to zero. The dust luminosity due to star formation could therefore in principle be up to a factor of two smaller if the spatial distribution of the emission is ignored. Given that the photometry reaches only to rest-frame $\mathrm{V}$ band, it remains possible to hide a very large stellar mass behind plausible values for the extinction $\left(A_{\mathrm{V}} \leq 6\right.$, as seen in other massive dusty galaxies $\frac{14 \sqrt{16} 52 \mid 53}{5}$ ). Considering the IRAC flux densities alone, we can calculate simple restframe mass-to-light ratios for the observed bands to see what masses could exist without relying

on the poorly-constrained CIGALE SED modeling. We use a stellar population synthesis code 54 55 to compute a stellar mass-to-light ratio under a range of assumptions: stellar ages of $0.1-0.8 \mathrm{Gyr}$ (from a reasonably "young" population to the approximate age of the universe at the time) and metallicity of $0.1-1 Z_{\odot}$, with no dust attenuation. The mass then ranges from $(2-10) \times 10^{10} M_{\odot}$ per $\mu \mathrm{Jy}$ of measured flux density. Taking the measured and demagnified flux density (averaged between the two wavelengths) of $0.5 \mu \mathrm{Jy}$, we find a stellar mass of $(1-5) \times 10^{10} M_{\odot}$ before correcting for extinction. If the extinction is as large as 5 magnitudes, the true stellar mass could be unphysically large ( $>10^{12} M_{\odot}$ ), demonstrating that we have no useful constraint without greater certainty about the reliability of the IRAC flux densities and/or more photometric data points.

\section{Galaxy and Halo Masses}

Figures 2 and 3 compile mass measurements for high-redshift galaxies discovered by various techniques. The galaxy sample primarily comprises galaxies identified through their luminous dust emission (DSFGs) and optically-identified quasars, which are typically the objects with the largest gas, dust, or stellar masses at these redshifts. At the very highest redshifts, where very few galaxies 
have been found, objects selected based on their ultraviolet emission are also included. The subsets of galaxies included in each figure overlap significantly but are not identical because not all of the requisite information is available for each source.

Dust Mass: Mass estimates are literature values unmodified from the original publications $4,15,56,62$ owing to the heterogeneity of the data available across the sample. The dust masses are generally derived from the FIR continuum emission, using one to several wavelengths. Differences between the cosmology assumed in this work and the past publications result in unimportant corrections and are ignored.

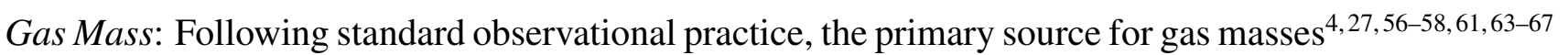
shown in Fig. 2(b) is measurement of the luminosity of rotational transitions of CO. The lowest available rotational transition is typically used and any translation between the observed transition and the $J=1-0$ line that is most commonly used as a molecular gas indicator is taken from the original source. Rather than accepting the varying coefficients for the conversion of CO luminosity to gas mass, we re-calculate all masses using a common value of $\alpha_{\mathrm{CO}}=1.0 M_{\odot}\left(\mathrm{K} \mathrm{km} \mathrm{s}^{-1} \mathrm{pc}^{2}\right)^{-1}$,

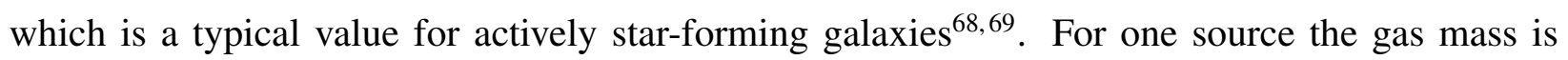
estimated through the star-formation surface density 59 .

Halo Mass: The halo masses of Fig. 3 are derived from the gas mass sample above. Each dark matter halo mass is represented using a range of values, starting with a conservative and hard lower limit found by dividing the measured gas mass by the universal baryon fraction ${ }^{10}$, $f_{\mathrm{b}}=0.19$. This lower limit ignores any baryonic mass that has been converted into stars and/or hot or cool atomic gas phases, which would increase the inferred halo mass. A more realistic, but still conservative, lower limit is represented by the top of the plotted symbols. Here we assume that the ratio of baryonic mass to halo mass is $M_{\mathrm{b}} / M_{\text {halo }}=0.05$. This value is a factor of $\sim 4$ less than the universal baryon fraction but still higher than the typical stellar-to-halo mass ratio inferred for halos of any mass and redshift via subhalo abundance matching 3 . Given that we do not expect high-mass galaxies such as SPT0311-58 to expel a large fraction of their molecular gas content $t^{70}$ or to later accrete dark matter without also accreting gas in proportion to the universal baryon fraction, it is reasonable to expect that the baryon-to-halo mass ratio should be less than this inferred upper limit on the stellar-to-halo mass ratio across all masses and redshifts.

HFLS3 and SPT0311-58 Masses: In the case of the two most distant DSFGs, HFLS3 ${ }^{4}$ and SPT0311-58, which have extensive FIR photometry and atomic/molecular line measurements, 
we also compute the gas mass using a joint continuum/line radiative transfer model described in Refs. 29 and 6. The mass for SPT0311-58 was computed in Ref. 6 without spatially-resolved (CO and $\left[\mathrm{C}_{\mathrm{I}}\right]$ ) line emission. For the purposes of Fig. 3 only the total gas mass of the two SPT0311-58 galaxies is important for estimating the halo mass. For Fig. 2 the dust mass is divided between the two sources according to the ratio of dust continuum emission in our resolved observations. The gas mass is similarly divided, though the velocity profile of the $\mathrm{CO}$ lines provides weak evidence that the molecular gas is concentrated in SPT0311-58W, which would increase the gas mass for this source by $15 \%$.

\section{Calculation of Halo Rareness}

Figure 3 demonstrates the "rareness" of SPT0311-58 by considering its position in the dark matter halo mass-redshift plane compared with other extreme high-redshift objects (DSFGs, quasars, and an LBG) believed to be hosted by massive dark matter halos. To quantify the rareness of these extreme objects we employ the method of Ref. 28, adopting their MATLAB script available at https://bitbucket.org/itrharrison/hh13-cluster-rareness and modified slightly

to extend the calculation to $z=10$. These authors describe how to properly compute $\left(z, M_{\text {halo }}\right)$ contours ("exclusion curves") above which the Poisson probability of such an object being detected in the standard $\Lambda \mathrm{CDM}$ cosmology is less than $\alpha(\alpha<1)$; the existence of a single object above such an exclusion curve is sufficient to rule out $\Lambda \mathrm{CDM}$ at the $100(1-\alpha) \%$ confidence level. In Fig. 3, we plot $1 \sigma$ exclusion curves (i.e., $\alpha=0.32$ ). Of the three different statistical measures of rareness that they propose, we employ the ' $>\nu$ ' measure, which quantifies the rareness according to the minimum height of the primordial density perturbation from which a halo of mass $M_{\text {halo }}$ and redshift $z$ could have formed, $\nu\left(M_{\text {halo }}, z\right) \propto\left(D_{+}(z) \sigma\left(M_{\text {halo }}\right)\right)^{-1}$, where $D_{+}(z)$ is the normalized linear growth function and $\sigma^{2}\left(M_{\text {halo }}\right)$ is the variance of the matter power spectrum smoothed on the comoving spatial scale that corresponds to the mass $M_{\text {halo }}$. This statistic is sensitive to changes in the $\Lambda \mathrm{CDM}$ initial conditions, such as primordial non-Gaussianity (which would lead to more high-mass dark matter halos at a given redshift than expected in the standard $\Lambda$ CDM cosmology). For the purposes of this calculation, we assume a $\Lambda$ CDM cosmology with parameters 10 $\left(\Omega_{m}=0.309, \Omega_{\Lambda}=0.691, h_{0}=0.677, \sigma_{8}=0.816\right)$ and employ the halo mass function of Ref. 71.

The $>\nu$ rareness statistic (and the corresponding exclusion curves) depends on the region of the $M_{\text {halo }}-z$ plane to which the survey is sensitive. We assume that the SPT sample of lensed DSFGs is complete for $z>1.5$. At lower redshift, the probability of lensing is strongly suppressed ${ }^{3072}$, which means that the galaxy (or galaxies) associated with a halo of 
$M_{\text {halo }} \gtrsim 10^{15} M_{\odot}$ (the $M_{\text {halo }}$ value of the exclusion curves for $z=1.5$ ) would have to have a very high intrinsic (i.e., unlensed) mm flux density $\left(S_{1.4} \gtrsim 20 \mathrm{mJy}\right)$ to be included in the sample. Because of the effects of "downsizing" (i.e., star formation is terminated at higher redshift in higher-mass galaxies than in lower-mass galaxies), it is unlikely that massive galaxies at $z<1.5$ would have sufficiently high IR luminosity to be detected by the SPT ${ }^{73}$. We furthermore assume that the survey is complete for $M_{\text {halo }}>10^{11} M_{\odot}$. The assumption that the sample is complete to $M_{\text {halo }}=10^{11} M_{\odot}$ is a conservative one because the galaxies hosted by such halos, which would have $M_{\mathrm{b}} \ll 10^{10} M_{\odot}$, are unlikely to be sufficiently luminous to be detected without being very strongly lensed ( $\mu \gtrsim 10$ ); erring on the side of overestimating the completeness yields a lower limit on the rareness. Substituting a minimum halo mass of, e.g., $10^{12} M_{\odot}$ would make the value of the $>\nu$ rareness statistic less than found for $10^{11} M_{\odot}$, i.e., SPT0311-58 would be inferred to be even rarer.

The total area from which the SPT DSFG sample was selected is $2500 \mathrm{deg}^{2}$. However, the fact that most of the SPT DSFGs are strongly lensed implies that the effective survey area is potentially much less than $2500 \mathrm{deg}^{2}$ because not only must a galaxy have a high intrinsic mm flux density to be included in the sample but also it must be gravitationally lensed by some factor in order to exceed the $S_{1.4 \mathrm{~mm}} \simeq 20 \mathrm{mJy}$ threshold for inclusion in redshift followup observations. Properly accounting for the effects of lensing on the sample completeness would require defining an effective survey area as a function of halo mass and redshift, $A_{\text {eff }}\left(M_{\text {halo }}, z\right)=2500 \operatorname{deg}^{2} P\left(\mu_{\text {min }} \mid M_{\text {halo }}, z\right)$, where $P\left(\mu \mid M_{\text {halo }}, z\right)$ is the probability of a galaxy hosted by a halo of mass $M_{\text {halo }}$ at redshift $z$ being lensed by a factor $\mu_{\min }$, the minimum magnification necessary for which a halo of mass $M_{\text {halo }}$ and redshift $z$ would be detectable. However, given the large uncertainties in determining such a function, we opt for a simpler approach. Instead, in Fig. 3, we plot exclusion curves for the full sky (dotted line); an area of $2500 \mathrm{deg}^{2}$ (dashed line), which corresponds to the assumption that all halos in the mass and redshift range specified above would be detected even if they were not lensed; and an area of $25 \mathrm{deg}^{2}$ (solid line), which corresponds to the assumption that the survey area corresponds to just the $\sim 1 \%$ of the SPT fields over which the magnification for sources at $z \gtrsim 1.5$ will be at least ${ }^{30.72} \mu=2$, like SPT0311-58.

Code Availability The lensing reconstruction for the ALMA data was initially performed with the visilens code available at https://github.com/jspilker/visilens. Pixelated reconstructions were performed using a proprietary code developed by a subset of the authors and additional non-authors, and we opt not to release this code in connection with this work. The rareness calculation was performed with publicly available code (https://bitbucket.org/ 
itrharrison/hh13-cluster-rareness). The image deblending for the Spitzer images used GALFIT (https://users.obs.carnegiescience.edu/peng/work/galfit/ galfit.html). The SED modeling used the CIGALE code (https:// cigale.lam.fr/), version 0.11.0. The photometric redshift of the lens galaxy was estimated with EAZY (https: //github.com/gbrammer/eazy-photoz).

Data Availability This paper makes use of the following ALMA data: ADS/JAO.ALMA\#2016.1.01293.S and ADS/JAO.ALMA\#2015.1.00504.S, available at http://almascience.org/aq?project_code=2015.1.00504.S and http://almascience.org/aq?project_code=2016.1.01293.S. The HST data are available online at the Mikulski Archive for Space Telescopes (MAST; https://archive.stsci.edu) under proposal ID 14740. Datasets analysed here are available from the corresponding author on reasonable request.

1. Springel, V. et al. Simulations of the formation, evolution and clustering of galaxies and quasars. Nature 435, 629-636 (2005).

2. Cole, S., Helly, J., Frenk, C. S. \& Parkinson, H. The statistical properties of $\Lambda$ cold dark matter halo formation. Mon. Not. R. Atron. Soc. 383, 546-556 (2008).

3. Behroozi, P. S., Wechsler, R. H. \& Conroy, C. The Average Star Formation Histories of Galaxies in Dark Matter Halos from z = 0-8. Astrophys. J. 770, 57 (2013).

4. Riechers, D. A. et al. A dust-obscured massive maximum-starburst galaxy at a redshift of 6.34. Nature 496, 329-333 (2013).

5. Vieira, J. D. et al. Dusty starburst galaxies in the early Universe as revealed by gravitational lensing. Nature 495, 344-347 (2013).

6. Strandet, M. L. et al. ISM Properties of a Massive Dusty Star-forming Galaxy Discovered at $z \sim 7$. Astrophys. J. Lett. 842, L15 (2017).

7. Cormier, D. et al. The Herschel Dwarf Galaxy Survey. I. Properties of the low-metallicity ISM from PACS spectroscopy. Astron. Astrophys. 578, A53 (2015).

8. Carlstrom, J. E. et al. The 10 Meter South Pole Telescope. Pub. Astron. Soc. Pac. 123, 568-581 (2011).

9. Mocanu, L. M. et al. Extragalactic Millimeter-wave Point-source Catalog, Number Counts and Statistics from $771 \mathrm{deg}^{2}$ of the SPT-SZ Survey. Astrophys. J. 779, 61 (2013). 
10. Planck Collaboration et al. Planck 2015 results. XIII. Cosmological parameters. Astron. Astrophys. 594, A13 (2016).

11. Casey, C. M., Narayanan, D. \& Cooray, A. Dusty star-forming galaxies at high redshift. Phys. Rep. 541, 45-161 (2014).

12. Hezaveh, Y. D. et al. Detection of Lensing Substructure Using ALMA Observations of the Dusty Galaxy SDP.81. Astrophys. J. 823, 37 (2016).

13. Mihos, J. C. \& Hernquist, L. Gasdynamics and Starbursts in Major Mergers. Astrophys. J. 464, 641 (1996).

14. Cooray, A. et al. HerMES: The Rest-frame UV Emission and a Lensing Model for the $\mathrm{z}=$ 6.34 Luminous Dusty Starburst Galaxy HFLS3. Astrophys. J. 790, 40 (2014).

15. Decarli, R. et al. Rapidly star-forming galaxies adjacent to quasars at redshifts exceeding 6. Nature 545, 457-461 (2017).

16. Casey, C. M. et al. Near-infrared MOSFIRE Spectra of Dusty Star-forming Galaxies at $0.2<$ $\mathrm{z}<4$. Astrophys. J. 840, 101 (2017).

17. Bouwens, R. J. et al. UV Luminosity Functions at Redshifts $\mathrm{z} \sim 4$ to $\mathrm{z} \sim 10$ : 10,000 Galaxies from HST Legacy Fields. Astrophys. J. 803, 34 (2015).

18. Ferkinhoff, C. et al. First Detection of the [O III] $88 \mu \mathrm{m}$ Line at High Redshifts: Characterizing the Starburst and Narrow-line Regions in Extreme Luminosity Systems. Astrophys. J. Lett. 714, L147-L151 (2010).

19. Brauher, J. R., Dale, D. A. \& Helou, G. A Compendium of Far-Infrared Line and Continuum Emission for 227 Galaxies Observed by the Infrared Space Observatory. Astrophys. J. Suppl. 178, 280-301 (2008).

20. Inoue, A. K. et al. Detection of an oxygen emission line from a high-redshift galaxy in the reionization epoch. Science 352, 1559-1562 (2016).

21. Lebouteiller, V. et al. Physical conditions in the gas phases of the giant H II region LMCN 11 unveiled by Herschel . I. Diffuse [C II] and [O III] emission in LMC-N 11B. Astron. Astrophys. 548, A91 (2012).

22. Díaz-Santos, T. et al. Explaining the [C II]157.7 $\mu \mathrm{m}$ Deficit in Luminous Infrared Galaxies - First Results from a Herschel/PACS Study of the GOALS Sample. Astrophys. J. 774, 68 (2013). 
23. Oteo, I. et al. Witnessing the Birth of the Red Sequence: ALMA High-resolution Imaging of [C II] and Dust in Two Interacting Ultra-red Starbursts at $\mathrm{z}=4.425$. Astrophys. J. 827, 34 (2016).

24. Spilker, J. S. et al. ALMA Imaging and Gravitational Lens Models of South Pole TelescopeSelected Dusty, Star-Forming Galaxies at High Redshifts. Astrophys. J. 826, 112 (2016).

25. Bolatto, A. D., Wolfire, M. \& Leroy, A. K. The CO-to- $\mathrm{H}_{2}$ Conversion Factor. Ann. Rev. Astron. Astrophys. 51, 207-268 (2013).

26. Bothwell, M. S. et al. A survey of molecular gas in luminous sub-millimetre galaxies. Mon. Not. R. Atron. Soc. 429, 3047-3067 (2013).

27. Aravena, M. et al. A survey of the cold molecular gas in gravitationally lensed star-forming galaxies at z 2. Mon. Not. R. Atron. Soc. 457, 4406-4420 (2016).

28. Harrison, I. \& Hotchkiss, S. A consistent approach to falsifying $\Lambda$ CDM with rare galaxy clusters. J. Cosmology Astropart. Phys. 7, 022 (2013).

29. Weiß, A. et al. Highly-excited CO emission in APM $08279+5255$ at z $=3.9$. Astron. Astrophys. 467, 955-969 (2007).

30. Weiß, A. et al. ALMA Redshifts of Millimeter-selected Galaxies from the SPT Survey: The Redshift Distribution of Dusty Star-forming Galaxies. Astrophys. J. 767, 88 (2013).

31. Strandet, M. L. et al. The Redshift Distribution of Dusty Star-forming Galaxies from the SPT Survey. Astrophys. J. 822, 80 (2016).

32. Hezaveh, Y. D. et al. ALMA Observations of SPT-discovered, Strongly Lensed, Dusty, Starforming Galaxies. Astrophys. J. 767, 132 (2013).

33. Fazio, G. G. et al. The Infrared Array Camera (IRAC) for the Spitzer Space Telescope. Astrophys. J. Suppl. 154, 10-17 (2004).

34. Ashby, M. L. N. et al. SEDS: The Spitzer Extended Deep Survey. Survey Design, Photometry, and Deep IRAC Source Counts. Astrophys. J. 769, 80 (2013).

35. Schuster, M. T., Marengo, M. \& Patten, B. M. IRACproc: a software suite for processing and analyzing Spitzer/IRAC data. In Society of Photo-Optical Instrumentation Engineers (SPIE) Conference Series, vol. 6270 of Society of Photo-Optical Instrumentation Engineers (SPIE) Conference Series (2006). 
36. Bertin, E. \& Arnouts, S. SExtractor: Software for source extraction. Astron. Astrophys. Suppl. 117, 393-404 (1996).

37. Hook, I. M. et al. The Gemini-North Multi-Object Spectrograph: Performance in Imaging, Long-Slit, and Multi-Object Spectroscopic Modes. Pub. Astron. Soc. Pac. 116, 425-440 (2004).

38. Eikenberry, S. et al. FLAMINGOS-2: the facility near-infrared wide-field imager and multiobject spectrograph for Gemini. In Ground-based and Airborne Instrumentation for Astronomy IV, vol. 8446 of Proc. SPIE, 84460I (2012).

39. Warner, C., Packham, C., Eikenberry, S. S. \& Gonzalez, A. GPUs and Python: A Recipe for Lightning-Fast Data Pipelines. In Ballester, P., Egret, D. \& Lorente, N. P. F. (eds.) Astronomical Data Analysis Software and Systems XXI, vol. 461 of Astronomical Society of the Pacific Conference Series, 53 (2012).

40. Warner, C., Eikenberry, S. S., Gonzalez, A. H. \& Packham, C. Redefining the Data Pipeline Using GPUs. In Friedel, D. N. (ed.) Astronomical Data Analysis Software and Systems XXII, vol. 475 of Astronomical Society of the Pacific Conference Series, 79 (2013).

41. Ma, J. et al. Stellar Masses and Star Formation Rates of Lensed, Dusty, Star-forming Galaxies from the SPT Survey. Astrophys. J. 812, 88 (2015).

42. Suyu, S. H., Marshall, P. J., Hobson, M. P. \& Blandford, R. D. A Bayesian analysis of regularized source inversions in gravitational lensing. Mon. Not. R. Atron. Soc. 371, 983-998 (2006).

43. Brammer, G. B., van Dokkum, P. G. \& Coppi, P. EAZY: A Fast, Public Photometric Redshift Code. Astrophys. J. 686, 1503-1513 (2008).

44. Burgarella, D., Buat, V. \& Iglesias-Páramo, J. Star formation and dust attenuation properties in galaxies from a statistical ultraviolet-to-far-infrared analysis. Mon. Not. R. Atron. Soc. 360, 1413-1425 (2005).

45. Noll, S. et al. Analysis of galaxy spectral energy distributions from far-UV to far-IR with CIGALE: studying a SINGS test sample. Astron. Astrophys. 507, 1793-1813 (2009).

46. Bruzual, G. \& Charlot, S. Stellar population synthesis at the resolution of 2003. Mon. Not. R. Atron. Soc. 344, 1000-1028 (2003). 
47. Chabrier, G. Galactic Stellar and Substellar Initial Mass Function. Pub. Astron. Soc. Pac. 115, 763-795 (2003).

48. Draine, B. T. \& Li, A. Infrared Emission from Interstellar Dust. IV. The Silicate-Graphite-PAH Model in the Post-Spitzer Era. Astrophys. J. 657, 810-837 (2007).

49. Calzetti, D. et al. The Dust Content and Opacity of Actively Star-forming Galaxies. Astrophys. J. 533, 682-695 (2000).

50. Leitherer, C., Li, I.-H., Calzetti, D. \& Heckman, T. M. Global Far-Ultraviolet (912-1800 Å) Properties of Star-forming Galaxies. Astrophys. J. Suppl. 140, 303-329 (2002).

51. Blain, A. W., Barnard, V. E. \& Chapman, S. C. Submillimetre and far-infrared spectral energy distributions of galaxies: the luminosity-temperature relation and consequences for photometric redshifts. Mon. Not. R. Atron. Soc. 338, 733-744 (2003).

52. Hopwood, R. et al. Spitzer Imaging of Herschel-atlas Gravitationally Lensed Submillimeter Sources. Astrophys. J. Lett. 728, L4 (2011).

53. Lo Faro, B. et al. The Complex Physics of Dusty Star-forming Galaxies at High Redshifts as Revealed by Herschel and Spitzer. Astrophys. J. 762, 108 (2013).

54. Conroy, C., Gunn, J. E. \& White, M. The Propagation of Uncertainties in Stellar Population Synthesis Modeling. I. The Relevance of Uncertain Aspects of Stellar Evolution and the Initial Mass Function to the Derived Physical Properties of Galaxies. Astrophys. J. 699, 486-506 (2009).

55. Conroy, C. \& Gunn, J. E. The Propagation of Uncertainties in Stellar Population Synthesis Modeling. III. Model Calibration, Comparison, and Evaluation. Astrophys. J. 712, 833-857 (2010).

56. Wang, R. et al. CO (2-1) Line Emission in Redshift 6 Quasar Host Galaxies. Astrophys. J. Lett. 739, L34 (2011).

57. Wang, R. et al. Far-infrared and Molecular CO Emission from the Host Galaxies of Faint Quasars at z 6. Astron. J. 142, 101 (2011).

58. Walter, F. et al. The intense starburst HDF 850.1 in a galaxy overdensity at $z \approx 5.2$ in the Hubble Deep Field. Nature 486, 233-236 (2012). 
59. Watson, D. et al. A dusty, normal galaxy in the epoch of reionization. Nature 519, 327-330 (2015).

60. Venemans, B. P. et al. Bright [C ii] and Dust Emission in Three z $>6.6$ Quasar Host Galaxies Observed by ALMA. Astrophys. J. 816, 37 (2016).

61. Venemans, B. et al. The compact, $\sim 1 \mathrm{kpc}$ host galaxy of a quasar at $\mathrm{z}=7.1$. ArXiv e-prints (2017).

62. Laporte, N. et al. Dust in the Reionization Era: ALMA Observations of a $\mathrm{z}=8.38$ Gravitationally Lensed Galaxy. Astrophys. J. Lett. 837, L21 (2017).

63. Walter, F. et al. Molecular gas in the host galaxy of a quasar at redshift $\mathrm{z}=6.42$. Nature 424, 406-408 (2003).

64. Riechers, D. A. et al. A Massive Molecular Gas Reservoir in the $\mathrm{z}=5.3$ Submillimeter Galaxy AzTEC-3. Astrophys. J. Lett. 720, L131-L136 (2010).

65. Wang, R. et al. Molecular Gas in z 6 Quasar Host Galaxies. Astrophys. J. 714, 699-712 (2010).

66. Wang, R. et al. Star Formation and Gas Kinematics of Quasar Host Galaxies at z 6: New Insights from ALMA. Astrophys. J. 773, 44 (2013).

67. Rawle, T. D. et al. [C II] and ${ }^{12} \mathrm{CO}(1-0)$ Emission Maps in HLSJ091828.6+514223: A Strongly Lensed Interacting System at z = 5.24. Astrophys. J. 783, 59 (2014).

68. Solomon, P. M. \& Vanden Bout, P. A. Molecular Gas at High Redshift. Ann. Rev. Astron. Astrophys. 43, 677-725 (2005).

69. Carilli, C. L. \& Walter, F. Cool Gas in High-Redshift Galaxies. Ann. Rev. Astron. Astrophys. 51, 105-161 (2013).

70. Hayward, C. C. \& Hopkins, P. F. How stellar feedback simultaneously regulates star formation and drives outflows. Mon. Not. R. Atron. Soc. 465, 1682-1698 (2017).

71. Tinker, J. et al. Toward a Halo Mass Function for Precision Cosmology: The Limits of Universality. Astrophys. J. 688, 709-728 (2008).

72. Hezaveh, Y. D. \& Holder, G. P. Effects of Strong Gravitational Lensing on Millimeter-wave Galaxy Number Counts. Astrophys. J. 734, 52-59 (2011). 
73. Miller, T. B., Hayward, C. C., Chapman, S. C. \& Behroozi, P. S. The bias of the submillimetre galaxy population: SMGs are poor tracers of the most-massive structures in the $\mathrm{z} \sim 2$ Universe. Mon. Not. R. Atron. Soc. 452, 878-883 (2015).

Acknowledgements ALMA is a partnership of ESO (representing its member states), NSF (USA) and NINS (Japan), together with NRC (Canada) and NSC and ASIAA (Taiwan), in cooperation with the Republic of Chile. The Joint ALMA Observatory is operated by ESO, AUI/NRAO and NAOJ. This work incorporates observations with the NASA/ESA Hubble Space Telescope, obtained at the Space Telescope Science Institute (STScI) operated by AURA, Inc. This work is also based in part on observations made with the Spitzer Space Telescope, which is operated by the Jet Propulsion Laboratory, California Institute of Technology under a contract with NASA. The SPT is supported by the National Science Foundation through grant PLR-1248097, with partial support through PHY-1125897, the Kavli Foundation and the Gordon and Betty Moore Foundation grant GBMF 947. Supporting observations were obtained at the Gemini Observatory, which is operated by the Association of Universities for Research in Astronomy, Inc., under a cooperative agreement with the NSF on behalf of the Gemini partnership: the National Science Foundation (United States), the National Research Council (Canada), CONICYT (Chile), Ministerio de Ciencia, Tecnología e Innovación Productiva (Argentina), and Ministério da Ciência, Tecnologia e Inovação (Brazil). D.P.M., J.S.S., J.D.V., K.C.L., and J.S. acknowledge support from the U.S. National Science Foundation under grant AST-1312950. D.P.M. was also partially supported by NASA through grant HST-GO-14740 from the Space Telescope Science Institute and K.C.L. was partially supported by SOSPA4-007 from the National Radio Astronomy Observatory. The Flatiron Institute is supported by the Simons Foundation. J.D.V. acknowledges support from an A. P. Sloan Foundation Fellowship.

Author Contributions D.P.M. proposed the ALMA [C II] and [O III] line observations and analyzed all ALMA data. J.S.S. performed the lens modeling. C.C.H. led the rareness analysis. M.L.N.A., M.B.B., S.C.C, A.H.G, J.M., K.M.R., and B.S. provided optical/infrared data reduction and deconvolution. K.A.P. and J.D.V. performed SED modeling of the sources and lens. A.W. performed joint dust/line modeling of high-redshift targets. D.P.M. wrote the manuscript. J.S.S., C.C.H., D.P.M., S.L., K.A.P., and J.D.V. prepared the figures. All authors discussed the results and provided comments on the paper. The authors are ordered alphabetically after J.D.V.

Author Information Reprints and permissions information is available at www.nature.com/reprints. The authors declare no competing financial interests. Readers are welcome to comment on the online version of the paper. Publishers note: Springer Nature remains neutral with regard to jurisdictional claims in published maps and institutional affiliations. Correspondence and requests for materials should be addressed to D.P.M. (dmarrone@email.arizona.edu). 


\section{Extended Data Table 1 | ALMA Observations}

\begin{tabular}{|c|c|c|c|c|c|c|c|c|}
\hline Date & $\begin{array}{l}\text { Frequency }{ }^{\mathrm{a}} \\
\quad(\mathrm{GHz})\end{array}$ & Antennas & $\begin{array}{c}\text { Resolution } \\
\text { (arcsec) }\end{array}$ & $\begin{array}{l}\text { Flux } \\
\text { Calibrator }\end{array}$ & $\begin{array}{l}\text { Phase } \\
\text { Calibrator }\end{array}$ & $\begin{array}{l}\text { PWV }^{\mathrm{b}} \\
(\mathrm{mm})\end{array}$ & $\begin{array}{c}\mathrm{t}_{\text {int }}^{\mathrm{c}} \\
(\mathrm{min})\end{array}$ & $\begin{array}{c}\text { Noise Level } \\
\text { ( } \mu \mathrm{Jy} / \text { beam })\end{array}$ \\
\hline B3 & & & $3.3 \times 3.5$ & & & & & 35 \\
\hline 2016-Jan-02 & 91.95 & 41 & $3.8 \times 3.9$ & Uranus & J0303-6211 & 1.8 & 1.2 & 65 \\
\hline 2015-Dec-28 & 95.69 & 34 & $3.2 \times 3.5$ & Uranus & J0309-6058 & 2.9 & 1.2 & 83 \\
\hline 2015-Dec-28 & 99.44 & 34 & $3.1 \times 3.4$ & Uranus & J0309-6058 & 2.8 & 1.2 & 77 \\
\hline 2015-Dec-28 & 103.19 & 34 & $3.0 \times 3.4$ & Uranus & J0309-6058 & 2.7 & 1.5 & 72 \\
\hline 2015-Dec-28 & 106.94 & 34 & $2.9 \times 3.3$ & Uranus & J0309-6058 & 2.8 & 1.0 & 95 \\
\hline \multicolumn{9}{|l|}{ B6 } \\
\hline 2016-Nov-03 & 233.65 & 45 & $0.25 \times 0.30$ & J0334-4008 & J0303-6211 & 0.5 & 32.4 & 24 \\
\hline \multicolumn{9}{|l|}{ B7 } \\
\hline 2016-Jun-04 & 343.48 & 41 & $0.31 \times 0.49$ & J2258-2758 & J0303-6211 & 0.8 & 6.5 & 12 \\
\hline B8 & & & $0.20 \times 0.30$ & & & & & 53 \\
\hline 2016-Nov-15 & 423.63 & 41 & & J0538-4405 & J0253-5441 & 0.8 & 11.4 & \\
\hline 2016-Nov-16 & 423.63 & 42 & & J0538-4405 & J0253-5441 & 0.5 & 33.7 & \\
\hline 2016-Nov-16 & 423.63 & 42 & & J0538-4405 & J0253-5441 & 0.4 & 33.7 & \\
\hline 2016-Nov-17 & 423.63 & 43 & & J0538-4405 & J0253-5441 & 0.3 & 33.7 & \\
\hline
\end{tabular}

Extended Data Table 2 | Optical//R Photometry

\begin{tabular}{llccc}
\hline Telescope & Instrument/Filter & Lens & SPT0311-58E & SPT0311-58W \\
\hline$H S T$ & ACS/F606W & $>27.05$ & $>28.11$ & $>27.08$ \\
$H S T$ & ACS/F775W & $>26.55$ & $>27.59$ & $>26.63$ \\
Gemini & GMOS $/ i^{\prime}$ & $25.00 \pm 0.20$ & & \\
Gemini & GMOS $/ z^{\prime}$ & $24.40 \pm 0.20$ & & \\
$H S T$ & WFC3/F125W & $23.06 \pm 0.16$ & $25.28 \pm 0.10$ & $>26.69$ \\
$H S T$ & WFC3/F160W & $22.76 \pm 0.15$ & $24.98 \pm 0.12$ & $>27.11$ \\
Gemini & FLAMINGOS/ $/ K_{s} 2.16 \mu \mathrm{m}$ & $22.42 \pm 0.13$ & $\ldots$ & $\ldots$ \\
Spitzer & IRAC/Ch1 3.6 $\mu \mathrm{m}$ & $21.40 \pm 0.14$ & $24.47 \pm 0.30$ & $(23.87 \pm 0.28)$ \\
Spitzer & IRAC/Ch2 $4.5 \mu \mathrm{m}$ & $21.63 \pm 0.13$ & $24.45 \pm 0.25$ & $(23.63 \pm 0.22)$
\end{tabular}

All data in apparent (i.e., not corrected for magnification) AB magnitudes. Limiting magnitudes reported as $1 \sigma$ values. The magnification estimates for the $E$ and $W$ sources are 1.3 and 2.1, respectively, as reported in Methods Section 6 IRAC photometry for SPT0311-58W is uncertain due to blending with the lens, as noted in Methods Section 5

\section{Extended Data Table 3 | Far-Infrared Photometry}

\begin{tabular}{lcccc}
\hline Telescope & Observed Wavelength & $S_{\nu}$ (east intrinsic) & $S_{\nu}$ (west intrinsic) & $S_{\nu}$ (total apparent) \\
\hline Herschel/SPIRE $^{\mathrm{a}}$ & $250 \mu \mathrm{m}$ & $1.9 \pm 0.6$ & $12.7 \pm 4.2$ & $29.0 \pm 8.0$ \\
Herschel/SPIRE $^{\mathrm{a}}$ & $350 \mu \mathrm{m}$ & $2.5 \pm 0.5$ & $16.6 \pm 2.9$ & $38.0 \pm 6.0$ \\
Herschel/SPIRE $^{\mathrm{a}}$ & $500 \mu \mathrm{m}$ & $3.5 \pm 0.6$ & $22.7 \pm 4.2$ & $52.0 \pm 8.0$ \\
ALMA/B8 $_{\text {ALMA/B7 }}^{710 \mu \mathrm{m}}$ & $3.1 \pm 0.2$ & $19.9 \pm 0.3$ & \\
ALMA/B6 & $869 \mu \mathrm{m}$ & $2.9 \pm 0.2$ & $15.9 \pm 0.25$ & \\
ALMA/B3 & $1.26 \mathrm{~mm}$ & $1.18 \pm 0.05$ & $9.77 \pm 0.15$ & \\
\hline \multicolumn{4}{c}{ Flux densities $\left(S_{\nu}\right)$ in mJy. } \\
\hline
\end{tabular}

a Herschel photometry does not spatially resolve the two components. See Methods Section6 for details. 

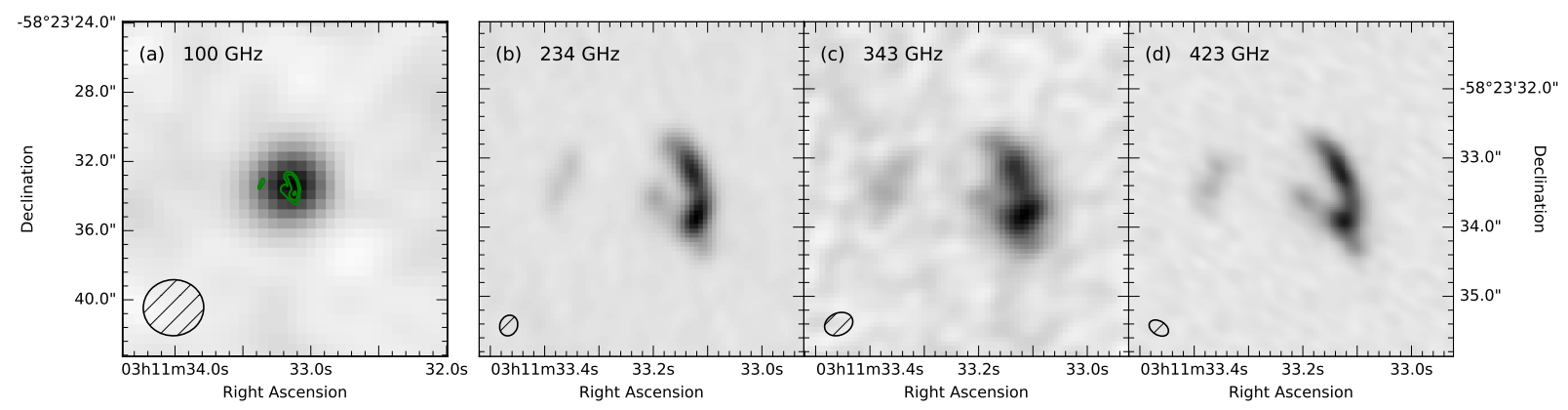

Extended Data Figure 1 | ALMA Continuum Images of SPT0311-58. From left to right, continuum images in ALMA bands 3, 6, 7, and 8, corresponding to rest-frame wavelengths of 380, 160,110 , and $90 \mu \mathrm{m}$, respectively. Note that the resolution in the first panel is a factor of roughly ten worse than the other images, and the displayed field of view is also larger by a factor of 4 . Contours at 10, 30, and 90\% of the image peak in band 6 are shown in the band 3 panel for scale. The ALMA synthesized beam (full-width-at-half-maximum) is represented as a hatched ellipse in the corner of each image.

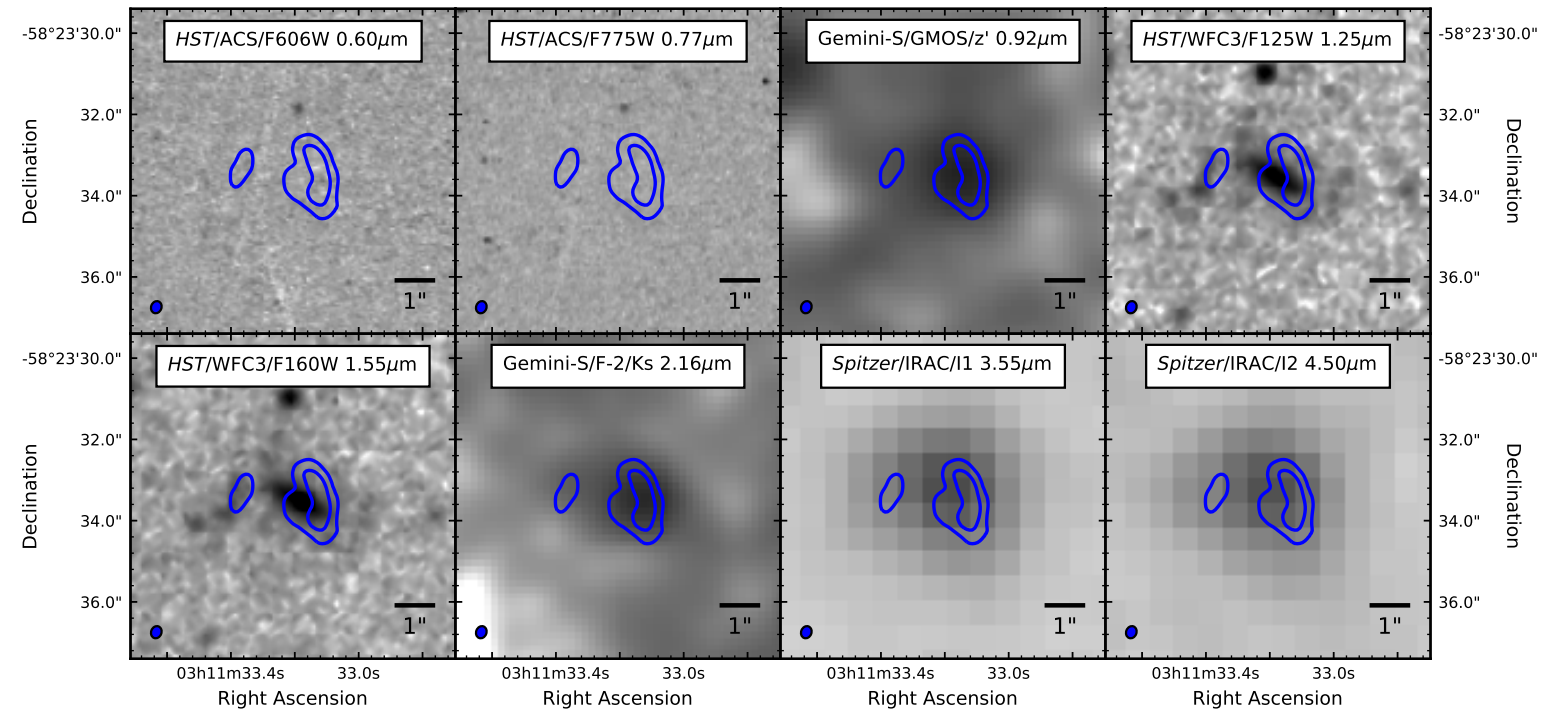

Extended Data Figure 2 Infrared and optical imaging of SPT0311-58. $8^{\prime \prime} \times 8^{\prime \prime}$ thumbnails of SPT0311-58 in the observed optical and infrared filters. ALMA band 6 continuum contours at 30 and $4 \%$ of the image peak are shown in blue along with the ALMA synthesized beam depicted as blue ellipse in the corner of each image. 


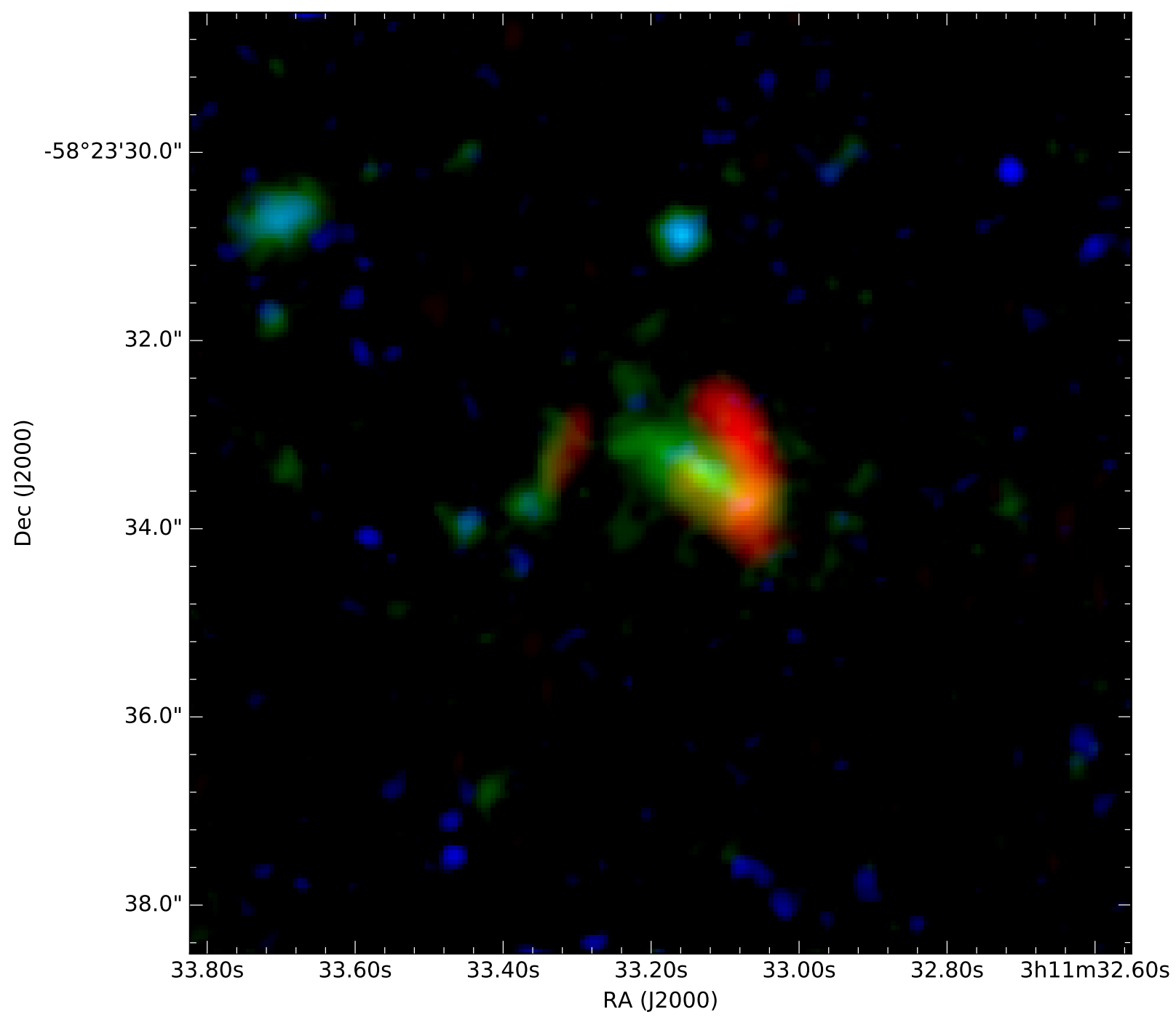

Extended Data Figure 3 | Optical, Infrared, and Millimeter image of SPT0311-58. The field around SPT0311-58 as seen with ALMA and HST at $1.3 \mathrm{~mm}$ (ALMA band 6; red), $1300 \mathrm{~nm}$ (combined Hubble/WFC3 F125W and F160W filters; green), and $700 \mathrm{~nm}$ (combined Hubble/ACS F606W and F775W filters; blue). For emission from $z=6.9$, no emission should be visible in the ACS filters due to the opacity of the neutral intergalactic medium, while the other filters correspond to rest-frame $160 \mathrm{~nm}$ and $160 \mu \mathrm{m}$. 


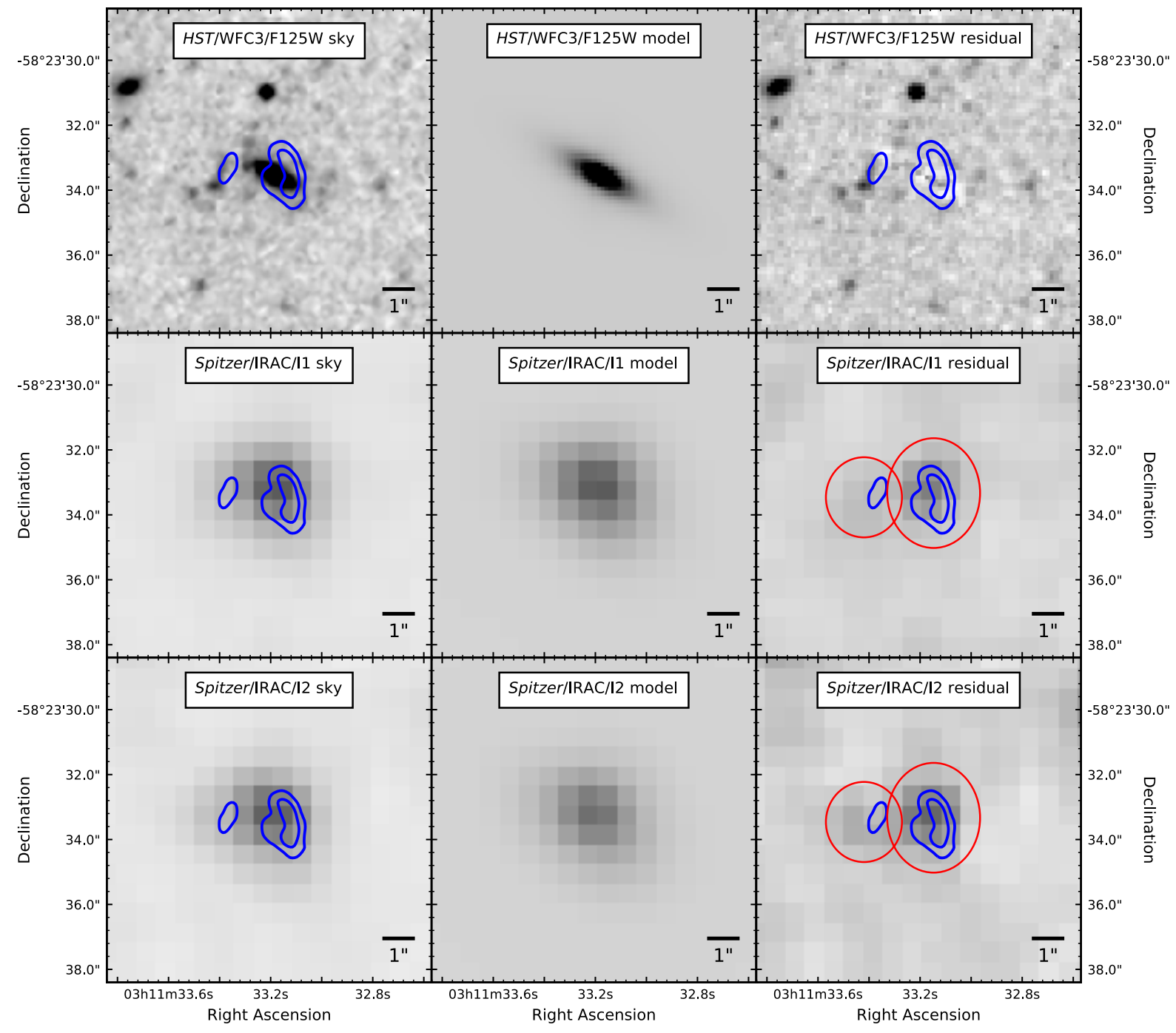

Extended Data Figure 4 | De-blending of the optical/infrared images. Left-right: sky image, model, and residual images. Top-bottom: Hubble/WFC3 F125W, Spitzer/IRAC $3.6 \mu \mathrm{m}$, and $4.5 \mu \mathrm{m}$ data. The ALMA band 6 contours are shown in the left and right columns, and the red circles in the right column show the photometric extraction regions for the Spitzer/IRAC images. 

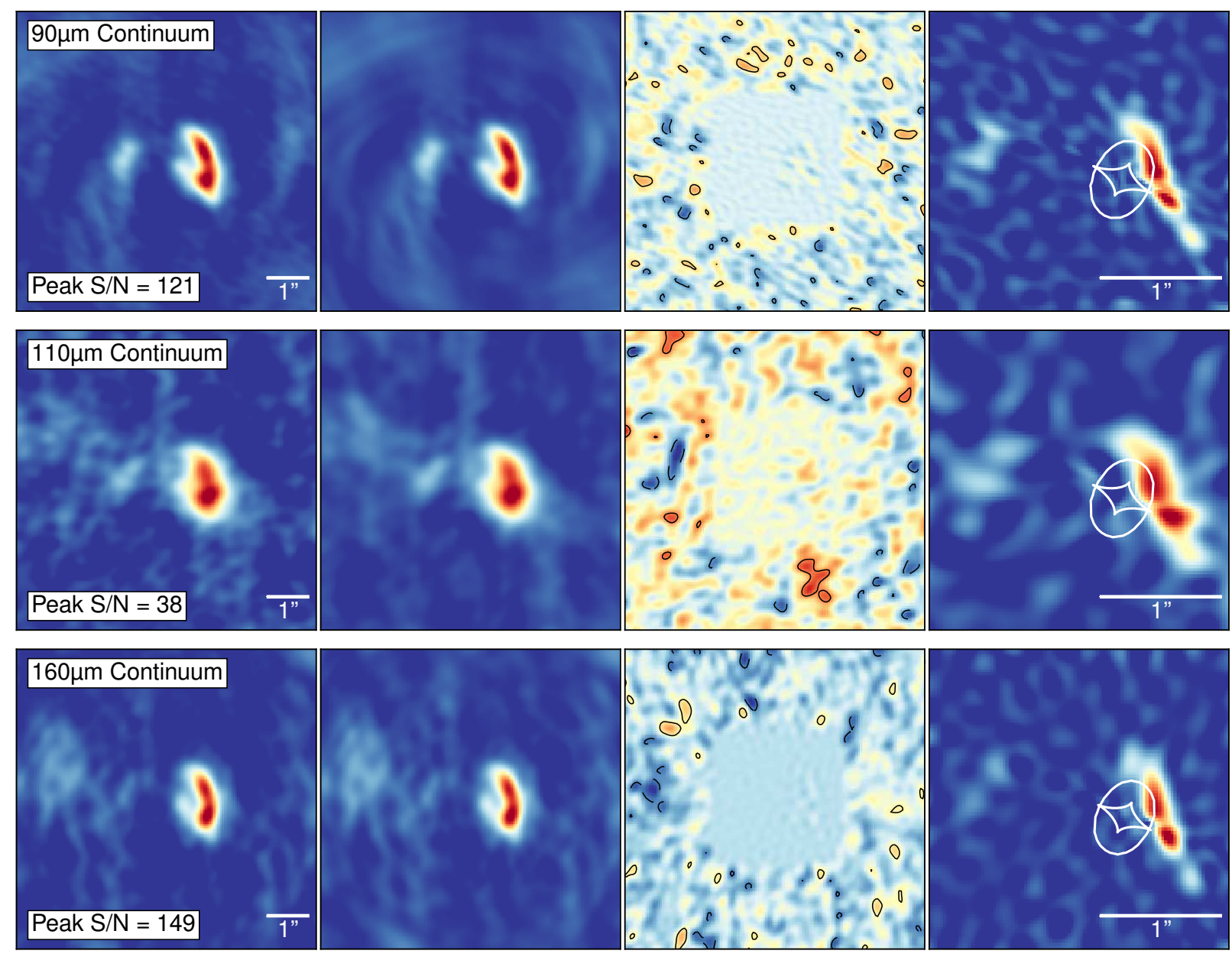

\section{Extended Data Figure 5 | Gravitational lensing model of the dust continuum emission in}

SPT0311-58. For each continuum wavelength for which we have suitable data, we reconstruct the source-plane emission as described in Section 6. For each wavelength, from left to right, we show the "dirty" (i.e., not deconvolved) image of the data, the dirty image of the model, the model residuals, and the source-plane reconstruction. Because the images of the data are not deconvolved, the apparently high-significance structure far from the object is due to sidelobes in the synthesized beam, and should be reproduced by the models. The image-plane region modeled is evident in the residuals, and results in the "noise" in the source-plane reconstructions. Contours in the residual panels are drawn in steps of $\pm 2 \sigma$. The lensing caustics are shown in each source-plane panel. The lens parameters are determined independently at 90 and $160 \mu \mathrm{m}$; at $110 \mu \mathrm{m}$ we adopt the parameters of the $160 \mu \mathrm{m}$ model. 


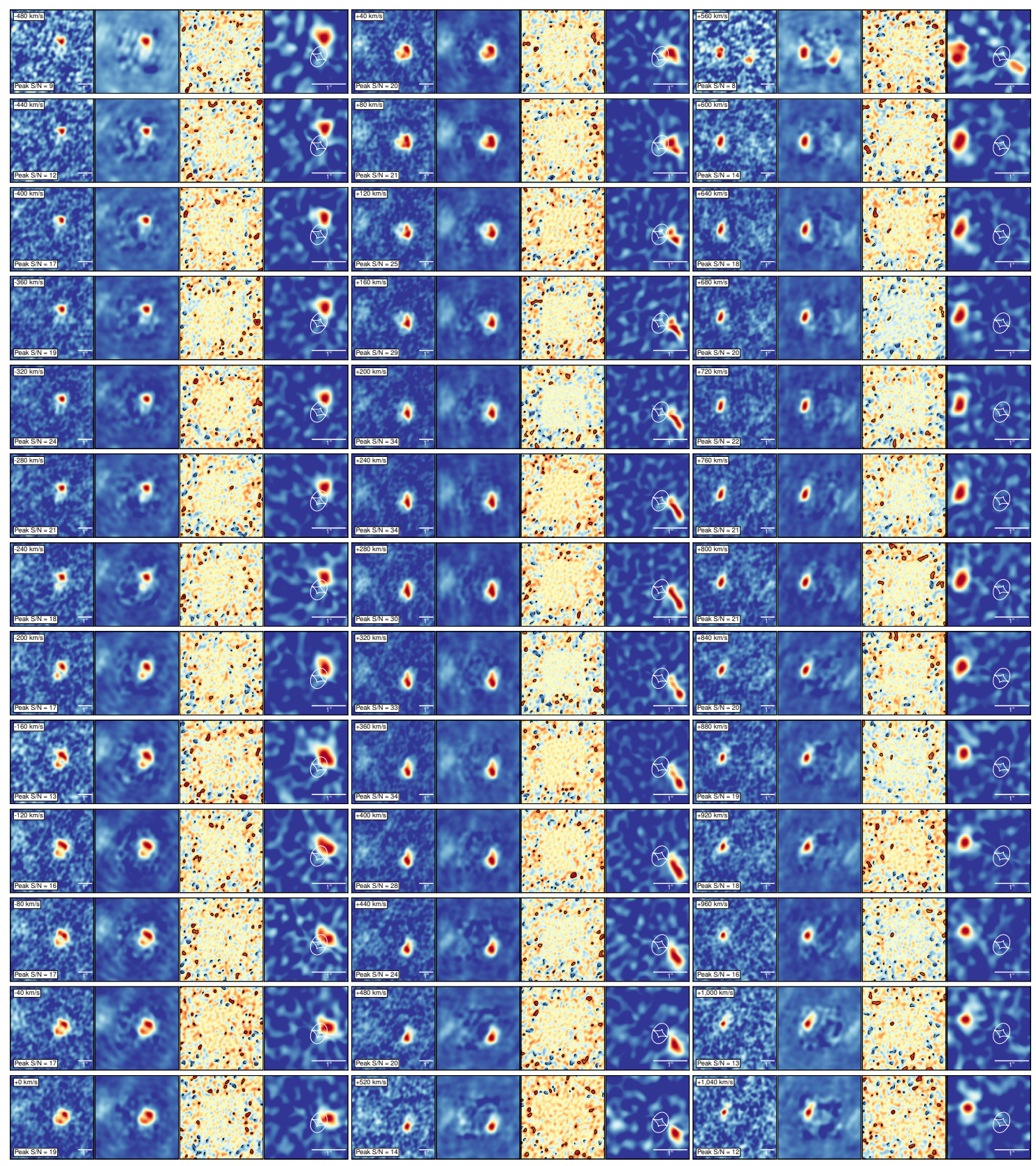

Extended Data Figure 6 | Gravitational lensing model of the [C $\mathrm{CI}_{\text {II }}$ line in SPT0311-58. For each $40 \mathrm{~km} \mathrm{~s}^{-1}$-wide channel, we reconstruct the source-plane emission using the lens parameters determined from fitting to the rest-frame $160 \mu \mathrm{m}$ (ALMA Band 6) continuum data (Section 6). We show for each channel, from left to right, the "dirty" (i.e., not deconvolved) image of the data, the dirty image of the model, the model residuals, and the source-plane reconstruction. Because the images of the data are not deconvolved, the apparently high-significance structure far from the object is due to sidelobes in the synthesized beam, and should be reproduced by the models. Contours in the residual panels are drawn in steps of $\pm 2 \sigma$. The lensing caustics are shown in each source-plane panel. 


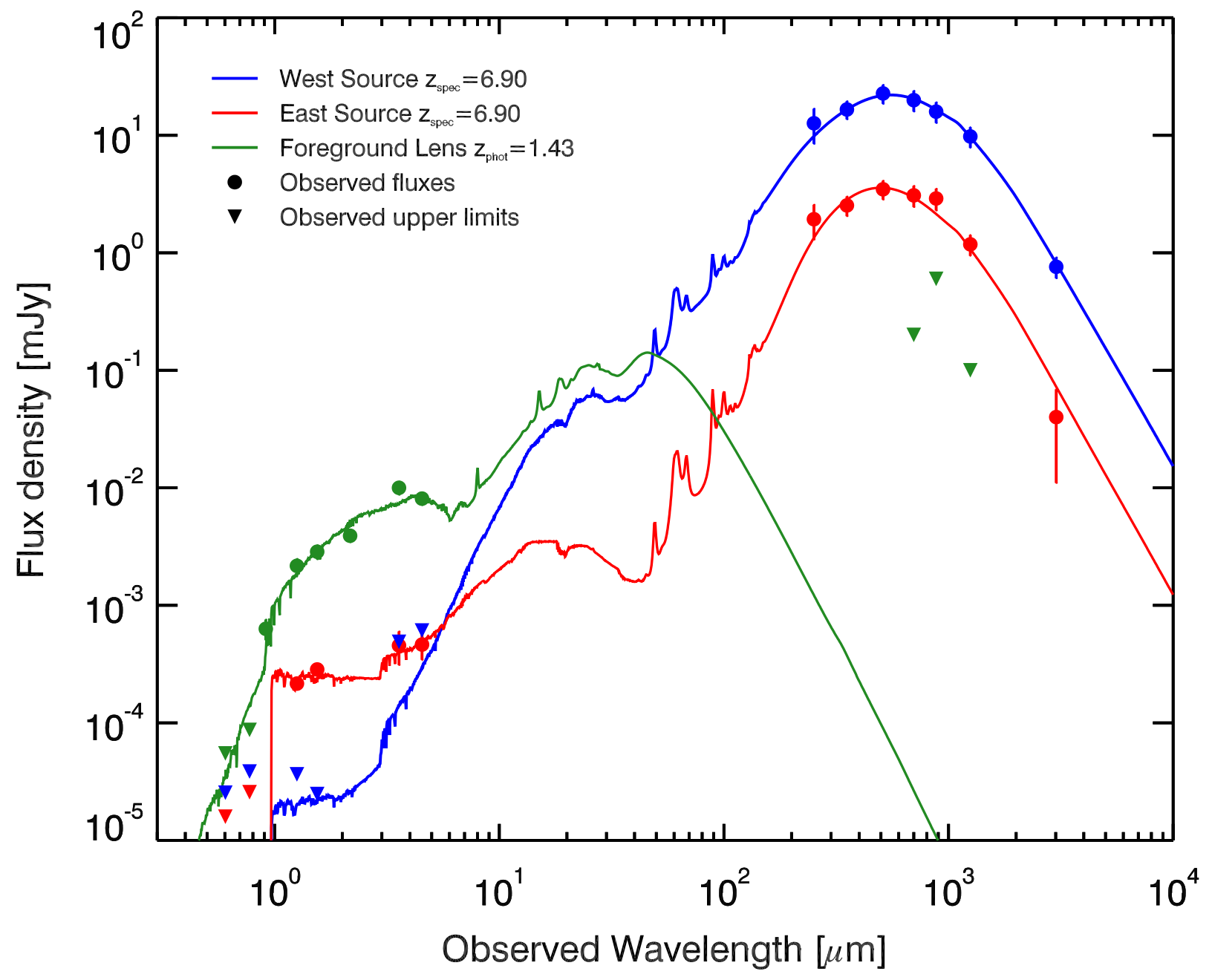

Extended Data Figure 7 | Optical to submillimeter spectral energy distribution modeling for SPT0311-58 $\mathbf{E}$ and $\mathbf{W}$ and the lensing galaxy. The photometric data in Extended Data Tables 2 and 3 for the three components at the position of SPT0311-58 are compared to the models determined using the CIGALE spectral energy distribution modeling code. The lens is modeled assuming a redshift of 1.43 , as estimated with the photometric redshift code EAZY. Upper limits are shown at the $1 \sigma$ threshold. 